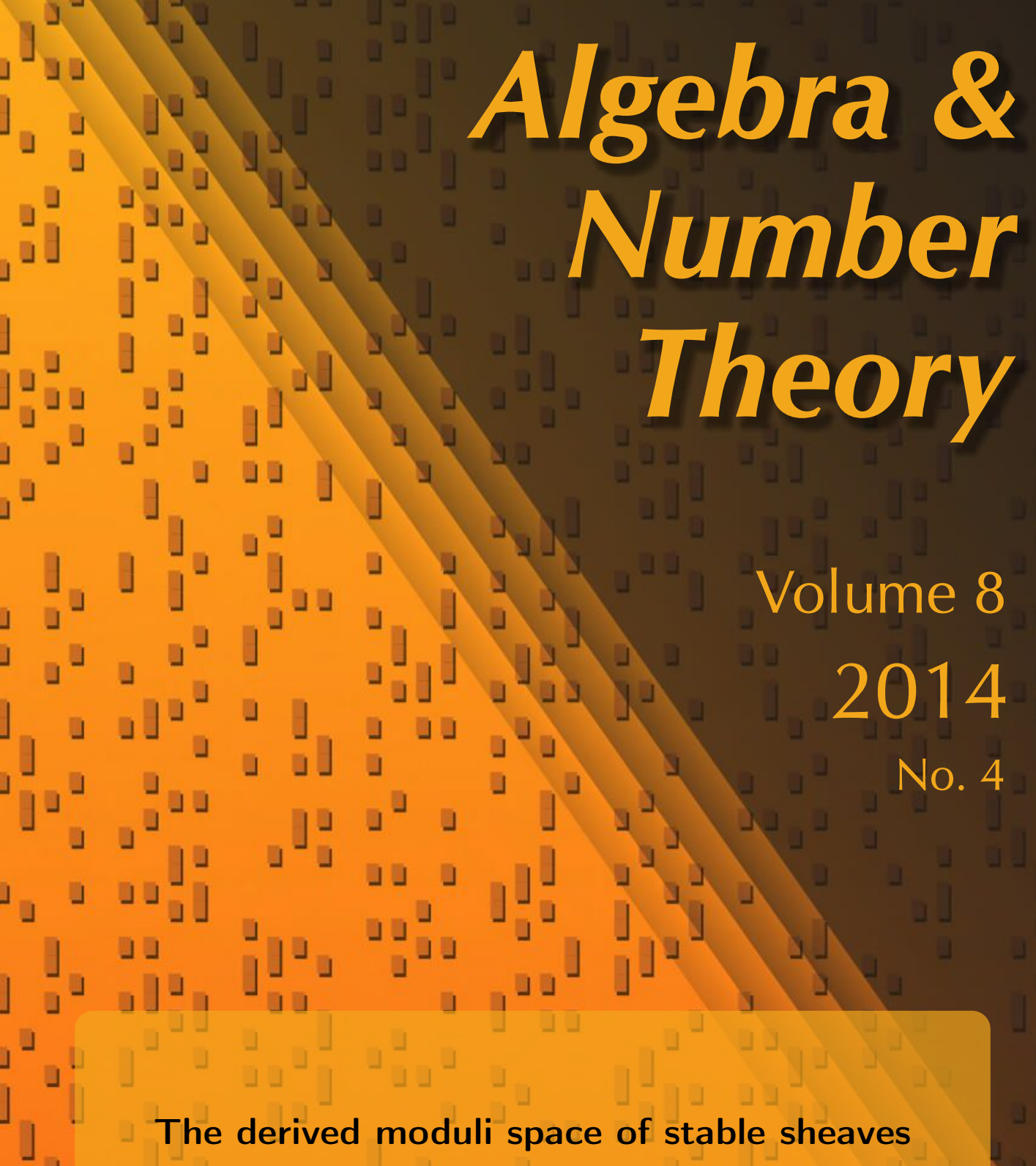

Kai Behrend, lonut Ciocan-Fontanine, Junho Hwang and Michael Rose

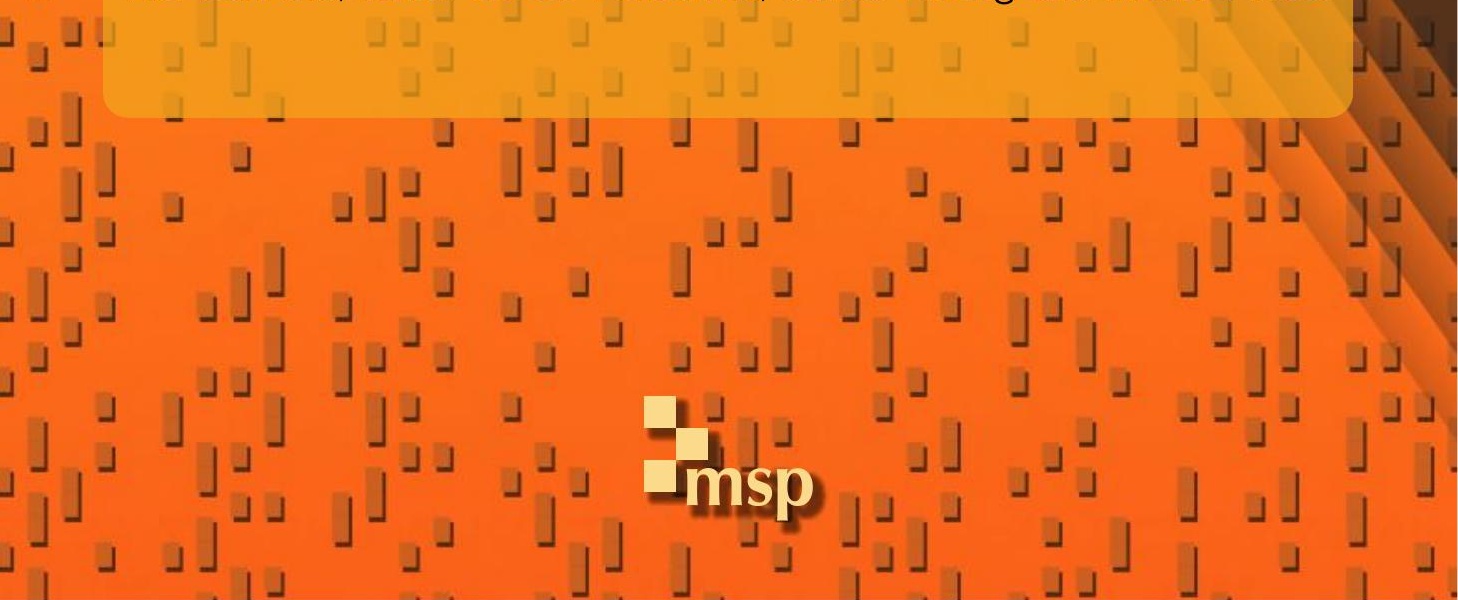




\title{
The derived moduli space of stable sheaves
}

\author{
Kai Behrend, lonut Ciocan-Fontanine, Junho Hwang and Michael Rose
}

\begin{abstract}
We construct the derived scheme of stable sheaves on a smooth projective variety via derived moduli of finite graded modules over a graded ring. We do this by dividing the derived scheme of actions of Ciocan-Fontanine and Kapranov by a suitable algebraic gauge group. We show that the natural notion of GIT stability for graded modules reproduces stability for sheaves.
\end{abstract}

Introduction

Index of notation

1. The derived scheme of simple graded modules 788

1A. The differential graded Lie algebra $L \quad 788$

1B. The moduli stack of $L \quad 791$

1C. The derived space of equivalence classes of simple modules 796

1D. The tangent complex 798

2. Stability 801

3. Moduli of sheaves 803

3A. The adjoint of the truncation functor $\quad 803$

3B. Open immersion 804

3C. Stable sheaves 806

3D. Moduli of sheaves 807

3E. An amplification 808

4. Derived moduli of sheaves 810

$\begin{array}{ll}\text { References } & 811\end{array}$

\section{Introduction}

For some years it has been a tenet of geometry that deformation theory problems are governed by differential graded Lie algebras. This leads to formal moduli being given, dually, by differential graded commutative algebras and gives rise to the derived geometry program. Usually, the expectation is that to solve a given global moduli problem with a differential graded Lie algebra, this differential graded Lie

MSC2010: 14D20.

Keywords: differential graded schemes, curved differential graded Lie algebras, stable sheaves . 
algebra will have to be infinite-dimensional and therefore will be ill-suited for algebraic geometry.

For example, gauge theory can be used to construct analytic moduli spaces of holomorphic vector bundles on a compact complex manifold $Y$. In the case when the bundles are topologically trivial, the differential graded Lie algebra is $A^{0, \bullet}\left(Y, M_{n}\right)$, the algebra of $C^{\infty}$-forms of type $(0, \bullet)$ with values in $n \times n$-matrices (or a suitable completion thereof). The differential is the Dolbeault differential, and the bracket is combined from wedge product of forms and commutator bracket of matrices. Almost complex structures are elements $x \in A^{0,1}\left(Y, M_{n}\right)$, and they are integrable if and only if they satisfy the Maurer-Cartan equation

$$
d x+\frac{1}{2}[x, x]=0 .
$$

Dividing the Maurer-Cartan locus by the gauge group $G=A^{0,0}\left(Y, \mathrm{GL}_{n}\right)$, we obtain the moduli space of topologically trivial holomorphic bundles.

One central observation of this paper is that there exists a finite-dimensional analogue of this construction for moduli of coherent sheaves on a smooth projective variety over $\mathbb{C}$. Derived moduli of sheaves have been constructed before (see [Ciocan-Fontanine and Kapranov 2001] or [Toën and Vaquié 2007]), but we believe it is a new observation that there is a finite-dimensional differential graded Lie algebra with an algebraic gauge group, solving this moduli problem globally. Simply by virtue of being the space of Maurer-Cartan elements in a differential graded Lie algebra up to gauge equivalence, the moduli space automatically comes with a derived, or differential graded, structure.

This construction also leads one immediately to the examination of geometric invariant theory (GIT) stability for this algebraic gauge group action. Thus, another result of this paper it that GIT stability for our algebraic gauge group action reproduces the standard notion of stability for sheaves.

Let $Y$ be a smooth projective variety with homogeneous coordinate ring $A$ and $\alpha(t) \in \mathbb{Q}[t]$ a numerical polynomial.

We present a construction of the derived moduli scheme of stable sheaves on $Y$ as a Geometric Invariant Theory quotient of the derived scheme of actions. The derived scheme of actions, $\Re A c t$, was introduced by Ciocan-Fontanine and Kapranov [2001] as an auxiliary tool in their construction of the derived scheme of quotients, $\mathfrak{R} Q u o t$.

The basic idea is to describe a coherent sheaf $\mathscr{F}$ on $Y$ with Hilbert polynomial $\alpha(t)$ in terms of the associated finite-dimensional graded $A$-module

$$
\Gamma_{[p, q]} \mathscr{F}=\bigoplus_{i=p}^{q} \Gamma(Y, \mathscr{F}(i)),
$$

with dimension vector $\left.\alpha\right|_{[p, q]}=(\alpha(p), \ldots, \alpha(q))$, for $q \gg p \gg 0$. In fact, for any open bounded family $\mathfrak{U}$ of sheaves with Hilbert polynomial $\alpha(t)$ on $Y$, there exist 
$q \gg p \gg 0$, such that

$$
\Gamma_{[p, q]}: \mathfrak{U} \longrightarrow\left(\begin{array}{l}
\text { graded } A \text {-modules in }[p, q] \\
\text { with dimension vector }\left.\alpha\right|_{[p, q]}
\end{array}\right)
$$

is an open embedding of moduli functors (i.e., of stacks).

We construct a finite-dimensional differential graded Lie algebra

$$
L=\bigoplus_{n=0}^{q-p} L^{n}
$$

together with an algebraic gauge group $G$ (the Lie algebra of $G$ is $L^{0}$ ), acting linearly on $L$, such that $M C(L) / G$, the quotient of the solution set of the Maurer-Cartan equation

$$
d x+\frac{1}{2}[x, x]=0, \quad x \in L^{1},
$$

by the gauge group, is equal to the set (or rather stack) of graded $A$-modules concentrated in degrees between $p$ and $q$ with dimension vector $\left.\alpha\right|_{[p, q]}$, up to isomorphism.

We do this by fixing a finite-dimensional graded vector space $V$ of dimension $\left.\alpha\right|_{[p, q]}$. Then the degree 1 part of our differential graded Lie algebra is essentially $L^{1}=\operatorname{Hom}_{\mathrm{gr}}\left(A\right.$, End $\left._{\mathbb{C}} V\right)$, the space of degree preserving $\mathbb{C}$-linear maps from $A$ to End $_{\mathbb{C}} V$, and the solutions to the Maurer-Cartan equation (1) turn out to be precisely the algebra maps $A \rightarrow \operatorname{End}_{\mathbb{C}} V$, that is, the structures of graded $A$-modules on $V$. Taking the quotient by the gauge group $G=\mathrm{GL}_{\mathrm{gr}}(V)$ of graded automorphisms of $V$ can be viewed as removing the choice of basis in $V$.

Equivalently, a family of $A$-modules can be viewed as a graded vector bundle of rank $\left.\alpha\right|_{[p, q]}$, that is, a $G$-torsor, endowed with an $A$-action. This approach to constructing (derived) moduli of $A$-modules in these two steps by first constructing moduli of vector bundles, that is, the stack $B G$, and then a relative (derived) scheme of actions over $B G$ is standard. For example, Toën and Vaquié [2007] use this method to construct moduli of derived category objects.

Our main interest lies in the derived scheme, obtained by restricting to stable objects (which are simple) and then removing the automorphism group (which is $\mathbb{C}^{*}$ ) by passing to the space underlying the $\mathbb{C}^{*}$-gerbe.

The quotient $L^{1} / G$ is an instance of a moduli space of quiver representations. The relevant quiver is directed, which implies that all points of $L^{1}$ are unstable for the action of $G$. Using standard techniques (as in [King 1994]), we modify the action of $G$ on $L^{1}$ by a (canonical choice of) character of $G$ to obtain a welldefined GIT problem. Thus we obtain a quasiprojective moduli space $M C(L)^{s} / / G$ of stable $A$-modules with a compactification $M C(L)^{s s} / / G$ consisting of semistable $A$-modules. The key result is the following: 
Theorem. For every bounded family $\mathfrak{U}$ of sheaves on $Y$ with Hilbert polynomial $\alpha(t)$, there exist $q \gg p \gg 0$ such that if $\mathscr{F}$ is a member of $\mathfrak{U}$, then $\mathscr{F}$ is a stable sheaf if and only if $\Gamma_{[p, q]} \mathscr{F}$ is GIT-stable.

This shows that usual (semi)stability as defined by Simpson [1994] is the natural notion of (semi)stability for sheaves induced from GIT-stability via our construction. Moreover, using the fact that semistable sheaves are bounded and satisfy the valuative criterion for properness, we see that the moduli space of semistable sheaves with Hilbert polynomial $\alpha(t)$ is a union of connected components of the projective scheme $M C(L)^{s s} / / G$ of semistable modules.

This gives a new construction of the moduli space of (semi)stable sheaves on a projective variety. One advantage of our approach over others, such as the classical Quot-scheme approach of [Simpson 1994] and [Huybrechts and Lehn 1997] or the Quiver approach of [Álvarez-Cónsul and King 2007], is that Equation (1) provides us with a rather explicit set of equations cutting out the moduli space.

We can also explicitly describe the image of the moduli space of stable sheaves inside the moduli space of $[p, q]$-graded $A$-modules. Namely, it is the scheme of stable modules whose truncation into an interval $\left[p^{\prime}, q\right]$, for suitable $p^{\prime}$ between $p$ and $q$, is also stable.

Since $M C(L) / G$ is the moduli space of a differential graded Lie algebra, it (or rather its stable locus) is automatically a differential graded scheme. It is naturally embedded into the smooth stack $L^{1} / G$ as the "spectrum" of a sheaf of differential graded algebras $\mathscr{R}$ on $L^{1} / G$, obtained from the algebra of functions on the affine supermanifold

$$
L[1]^{\geq 0}
$$

with its induced derivation by descending to the $G$-quotient. It is this differential graded scheme structure on $M C(L) / G$ which we refer to as a derived scheme.

A derived scheme comes with higher obstruction spaces at every point. In our case, the higher obstruction spaces at the sheaf $\mathscr{F}$, or the corresponding point $M=\Gamma_{[p, q]} \mathscr{F}^{\mathscr{F}}$ of $M C(L) / G$, are given by

$$
\operatorname{Ext}_{\mathscr{O}_{Y}}^{i}(\mathscr{F}, \mathscr{F})=\operatorname{Ext}_{A}^{i}(M, M) .
$$

The corresponding virtual fundamental class (see [Behrend and Fantechi 1997] and [Ciocan-Fontanine and Kapranov 2009]) is thus the one giving rise to DonaldsonThomas invariants [Thomas 2000] if $Y$ is Calabi-Yau.

The differential graded Lie algebra $L$ is essentially the degree preserving part of the Hochschild cochain complex

$$
L^{n}=\operatorname{Hom}_{\mathbb{C}}\left(A^{\otimes n}, \operatorname{End}_{\mathbb{C}} V\right)
$$


of the graded ring $A$ with values in the graded bimodule $\operatorname{End}_{\mathbb{C}} V$, where $V$ is a finitedimensional graded $A$-module in degrees from $p$ to $q$ with dimension vector $\left.\alpha\right|_{[p, a]}$ together with its natural Lie bracket induced from the commutator bracket in End $V$.

Outline. In Section 1, we construct the derived scheme of finite-dimensional graded $A$-modules with fixed dimension vector. This works for any algebra over $\mathbb{C}$; in particular, there is no need for commutativity of $A$. The main purpose of this section is to carefully describe the various differential graded schemes and stacks we construct, and to do this as explicitly as possible in terms of our finite-dimensional differential graded Lie algebra with its gauge group. We hope the introduction of bundles of curved differential graded Lie algebras will clarify the global geometric objects described infinitesimally by differential graded Lie algebras. We also advocate the use of Maurer-Cartan equations as a convenient way to package higher structures, in particular, $A_{\infty}$-module structures.

Section 2 is devoted to the study of the GIT problem given by the action of the gauge group $G$ on the space $L^{1}$. In particular, we construct quasiprojective derived moduli spaces of equivalence classes of stable finite graded $A$-modules of given dimension vector. We hope there will be applications in noncommutative geometry.

In Section 3 we introduce our projective scheme $Y$ and consider the case where our graded ring $A$ is the homogeneous coordinate ring of $Y$. We compare the stability notions for sheaves on $Y$ and for graded $A$-modules. We prove the above theorem and the amplification mentioned.

Finally, in Section 4, we write down the derived moduli problem for sheaves on $Y$, which is solved by our differential graded scheme. This is the only place where we need $Y$ to be smooth. The reason we need smoothness is to assure that for a coherent sheaf $\mathscr{F}$ on $Y$, the spaces $\operatorname{Ext}_{\mathscr{O}_{Y}}^{i}(\mathscr{F}, \mathscr{F})$ vanish for sufficiently large $i$.

Derived geometry. For us, derived geometry is the geometry of differential graded schemes. We make a few informal remarks here. For more detailed expositions of derived geometry, see Toën-Vezzosi [2004; 2005; 2008] or Lurie [2009].

A differential graded scheme is a pair $\left(T, \mathscr{R}_{T}\right)$, where $T$ is a scheme and $\mathscr{R}_{T}$ is a sheaf of differential graded $\mathbb{C}$-algebras (without restriction on the grading) on $T$, endowed with a structure morphism of sheaves of algebras $\mathrm{O}_{T} \rightarrow \mathscr{R}_{T}^{0}$.

It is natural to require (and we make it part of the definition) that all differential graded schemes $\left(T, \mathscr{R}_{T}\right)$ satisfy $\mathscr{O}_{T}=\mathscr{L}^{0}\left(\mathscr{R}_{T}\right)$, where $\mathscr{L}^{0}\left(\mathscr{R}_{T}\right)=\operatorname{ker}\left(d: \mathscr{R}_{T}^{0} \rightarrow \mathscr{R}_{T}^{1}\right)$ is the sheaf of 0 -cycles in $\mathscr{R}_{T}$. This implies that $\mathscr{R}_{T}$ is a sheaf of differential graded $\mathcal{O}_{T}$-algebras. Then a morphism of differential graded schemes $\left(T, \mathscr{R}_{T}\right) \rightarrow\left(M, \mathscr{R}_{M}\right)$ is a pair $(\phi, \mu)$, where $\phi: T \rightarrow M$ is a morphism of schemes, and $\mu: \phi^{*} \mathscr{R}_{M} \rightarrow \mathscr{R}_{T}$ is a morphism of sheaves of differential graded $\mathrm{O}_{T}$-algebras.

The classical scheme associated to a differential graded scheme $\left(T, \mathscr{R}_{T}\right)$ is the closed subscheme of $T$ given by $\pi_{0}\left(T, \mathscr{R}_{T}\right)=\operatorname{Spec}_{\mathscr{O}_{T}} h^{0}\left(\mathscr{R}_{T}\right)$. 
A differential graded scheme is affine if it comes from a differential graded algebra which is free as a graded algebra, on a finite set of generators, all in nonpositive degree.

Differential graded schemes form a category. (One may replace morphisms by germs of morphisms, defined in suitable neighborhoods of the classical loci.) This category is enriched over simplicial sets: the $n$-simplices in $\operatorname{Hom}(X, Y)$ are the morphisms $X \times \Delta_{n} \rightarrow Y$, where $\Delta_{n}$ is the differential graded scheme (which is not affine) corresponding to the differential graded algebra of algebraic differential forms on the algebraic $n$-simplex.

The category of differential graded schemes also has a natural topology: the étale topology, in which a family $U_{i} \rightarrow U$ is a covering family if $\pi_{0}\left(U_{i}\right) \rightarrow \pi_{0}(U)$ is a covering family in the usual étale topology, and every $U_{i} \rightarrow U$ is an étale morphism, which means that $\pi_{0}\left(U_{i}\right) \rightarrow \pi_{0}(U)$ is étale in the usual sense, and

$$
h^{r}\left(\mathscr{R}_{U_{i}}\right)=h^{r}\left(\mathscr{R}_{U}\right) \otimes_{h^{0}\left(\Re_{U}\right)} h^{0}\left(\mathscr{R}_{U_{i}}\right) \quad \text { for all } r .
$$

A morphism of differential graded schemes is a quasiisomorphism if it is étale, and induces an isomorphism on $\pi_{0}$.

In analogy with the definition of algebraic spaces, one can define a derived scheme (or space) to be a simplicial presheaf $X$ on the category of differential graded schemes satisfying two properties:

(i) (sheaf property) For every hypercover $U_{\bullet} \rightarrow U$, the map

$$
X(U) \rightarrow \operatorname{hocolim} X\left(U_{\bullet}\right)
$$

is a weak equivalence.

(ii) (locally affine property) $X$ is étale locally weakly equivalent to a presheaf represented by an affine differential graded scheme.

The simplicial category of derived schemes localizes the differential graded schemes at the quasiisomorphisms.

A particularly nice class of differential graded schemes comes from bundles of curved differential graded Lie algebras on smooth schemes (see the beginning of Section 1B). Our main object of study, $\mathfrak{R} \tilde{\mathfrak{M}}_{\alpha}^{\mathrm{sp}}(A)=\left(\tilde{\mathfrak{M}}^{\mathrm{sp}}, \mathscr{R}\right)$ is of this form.

We find it plausible (this will be proved elsewhere) that differential graded schemes coming from bundles of curved differential graded Lie algebras represent simplicial presheaves satisfying the above two properties (at least if we restrict the underlying base category to affine objects). Therefore, the moduli functor represented by such a "nice" differential graded scheme, in the derived world, would be given directly by the functor it represents over the category of differential graded schemes as defined here. This is the moduli functor we examine. 


\section{Index of notation}

A A graded ring.

$\mathfrak{m}$ The maximal ideal of positive degree elements in $A$.

$\alpha$ Depending on the context, either a numerical polynomial $\alpha(t) \in$ $\mathbb{Q}[t]$ or a dimension vector $\left(\alpha_{p}, \ldots, \alpha_{q}\right)$.

$V \quad$ A graded vector space of dimension $\alpha=\left(\alpha_{p}, \ldots, \alpha_{q}\right)$.

$L \quad L^{n}=\operatorname{Hom}_{\mathrm{gr}}\left(\mathfrak{m}^{\otimes n}\right.$, End $\left.V\right)$, the differential graded Lie algebra; see Section $1 \mathrm{~A}$.

$M \quad$ The scheme $L^{1}$.

$X$ The Maurer-Cartan locus in $M$.

$\mathscr{R}_{M}$ The sheaf of differential graded algebras on $M$; see Section $1 \mathrm{~B}$.

$\operatorname{Act}_{\mathrm{gr}}(A, V)$ The scheme $X$, when it is viewed as representing the scheme of graded actions of $A$ on $V$.

$\mathfrak{R A c t}_{\mathrm{gr}}(A, V)$ The differential graded scheme $\left(M, \mathscr{R}_{M}\right)$, which is the derived scheme of actions.

$G \quad$ The gauge group $G=\prod_{i=p}^{q} \operatorname{GL}\left(V_{i}\right)$.

$\Delta$ The one-parameter subgroup of scalars in $G$.

$\widetilde{G}$ The quotient group $G / \Delta$.

$\mathfrak{M}$ The quotient stack $[M / G]$.

$\widetilde{\mathfrak{M}}$ The quotient stack $[M / \widetilde{G}]$.

$\tilde{\mathfrak{M}}^{\mathrm{sp}}$ The open substack of $\tilde{\mathfrak{M}}$, which is an algebraic space.

$\mathfrak{X}$ The Maurer-Cartan locus in $\mathfrak{M}$.

$\widetilde{\mathfrak{X}}$ The Maurer-Cartan locus in $\widetilde{\mathfrak{M}}$.

$\mathfrak{M o d}_{\alpha}(A) \quad$ The algebraic stack $\mathfrak{X}$, when it is viewed as the stack of graded $A$-modules of dimension $\alpha$.

$\mathfrak{R M O d}_{\alpha}(A) \quad$ The differential graded stack $\left(\mathfrak{M}, \mathscr{R}_{\mathfrak{M}}\right)$, which is the derived stack of graded modules.

$\tilde{\mathfrak{M}}_{\alpha}^{\mathrm{sp}}(A) \quad$ The algebraic space $\widetilde{\mathfrak{X}}$, when viewed as the space of equivalence classes of simple graded modules.

$\mathfrak{R} \tilde{\mathfrak{M}}_{\alpha}^{\mathrm{sp}}(A) \quad$ The differential graded algebraic space $\left(\tilde{\mathfrak{M}}^{\mathrm{sp}}, \mathscr{R}\right)$, which is the derived space of equivalence classes of simple modules.

$\mathfrak{R} \tilde{M}_{\alpha}^{s}(A)$ The stable locus inside $\mathfrak{R} \tilde{\mathfrak{M}}_{\alpha}^{\mathrm{sp}}(A)$.

$\mathfrak{R} \tilde{M}_{\text {Tod }}^{\text {sp }}\left(O_{Y}\right)$ The functor of equivalence classes of simple families of coherent sheaves on $Y$ with Hilbert polynomial $\alpha(t)$ parametrized by differential graded schemes.

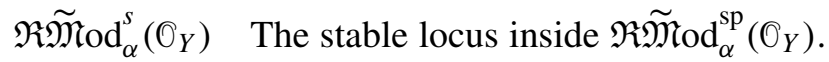


Notation and conventions. We work over a field of characteristic zero, which we shall denote by $\mathbb{C}$. All tensor products are over $\mathbb{C}$, unless indicated otherwise. All our differential graded algebras (and sheaves thereof), are graded commutative with unit.

Cohomology sheaves (of a complex of sheaves $\mathscr{C}^{\bullet}$ ) we usually denote by $h^{i}(\mathscr{E})$.

\section{The derived scheme of simple graded modules}

Let $A$ be a unital graded $\mathbb{C}$-algebra, not necessarily commutative, which is all in nonnegative degrees, and such that each graded piece is finite-dimensional. Moreover, we assume that the degree zero piece is one-dimensional, hence equal to $\mathbb{C}$. We denote by $\mathfrak{m}$ the ideal of elements of positive degree in $A$. Note that $\mathfrak{m}$ is a positively graded algebra without unit. We refer to the grading on $A$ as the internal or projective grading if there is a fear of confusion. We indicate this grading with lower indices.

Our main example of interest is that $A$ is the homogeneous coordinate ring of a projective variety over $\mathbb{C}$.

A graded $A$-module is the same thing as a graded $\mathfrak{m}$-module. The advantage of working with $\mathfrak{m}$ is that there is only one module axiom: associativity.

1A. The differential graded Lie algebra $L$. Let $V$ be a graded and finite-dimensional vector space

$$
V=\bigoplus_{i=p}^{q} V_{i} .
$$

By End $V$ we denote the algebra of $\mathbb{C}$-linear endomorphisms of $V$. It inherits a grading from $V$. Only $\operatorname{End}_{i} V$ in the range $i \in[p-q, q-p]$ are nonzero.

We denote the dimension vector of $V$ by

$$
\alpha=\left(\alpha_{p}, \ldots, \alpha_{q}\right)=\left(\operatorname{dim} V_{p}, \ldots, \operatorname{dim} V_{q}\right) .
$$

The graded vector space. We consider

$$
L^{n}=\operatorname{Hom}_{\mathrm{gr}}\left(\mathfrak{m}^{\otimes n}, \text { End } V\right),
$$

the vector space of degree-preserving $\mathbb{C}$-linear maps $\mu: \mathfrak{m}^{\otimes n} \rightarrow$ End $V$, and

$$
L=\bigoplus_{n=0}^{\infty} L^{n}
$$

Thus, $L^{0}=\operatorname{End}_{\mathrm{gr}} V$ and $L^{1}=\operatorname{Hom}_{\mathrm{gr}}(\mathfrak{m}$, End $V)$. We write elements $\mu \in L^{n}$ as multilinear maps $\mathfrak{m}^{\times n} \rightarrow$ End $V$. To distinguish the grading on $L$ from the projective grading, we may sometimes refer to it as the external grading. It is always indicated by upper indices. 
Note that every $L^{n}$ is finite-dimensional and that $L^{n}=0$, unless $n$ is in the range $n \in[0, q-p]$ because $\mathfrak{m}$ is positively graded.

Each $L^{n}$ is bigraded projectively:

$$
L^{n}=\bigoplus_{\substack{q \geq i \geq j \geq p \\ i-j \geq n}} L_{i j}^{n}
$$

where

$$
L_{i j}^{n}=\operatorname{Hom}\left(\left(\mathfrak{m}^{\otimes n}\right)_{i-j}, \operatorname{Hom}\left(V_{j}, V_{i}\right)\right) .
$$

For $n=0$, this simplifies to

$$
L^{0}=\bigoplus_{i=p}^{q} L_{i i}^{0}, \quad L_{i i}^{0}=\operatorname{Hom}\left(V_{i}, V_{i}\right)
$$

and for $n=1$, we can write

$$
L^{1}=\bigoplus_{q \geq i>j \geq p} L_{i j}^{1}, \quad L_{i j}^{1}=\operatorname{Hom}\left(\mathfrak{m}_{i-j}, \operatorname{Hom}\left(V_{j}, V_{i}\right)\right)
$$

We say that $L^{0}$ is diagonal, and $L^{1}$ is strictly lower triangular. The higher $L^{n}$ are restricted to successively smaller southwest corners.

The gauge group. We let $G=\mathrm{GL}_{\mathrm{gr}}(V)$ be the group of degree-preserving linear automorphisms of $V$ and call it the gauge group. Of course, $L^{0}$ is the Lie algebra of $G$. The gauge group is graded:

$$
G=\prod_{i=p}^{q} G_{i}, \quad G_{i}=\operatorname{GL}\left(V_{i}\right) .
$$

It acts, from the left, via conjugation on $L$. More precisely, for $g \in G$ and $\mu \in L^{n}$, we have

$$
(g \cdot \mu)\left(a_{1}, \ldots, a_{n}\right)=g \circ \mu\left(a_{1}, \ldots, a_{n}\right) \circ g^{-1} .
$$

The action of $G$ on $L^{n}$ preserves the double grading: if $g=\left(g_{p}, \ldots, g_{q}\right)$ and $\mu \in L^{n}$, then

$$
(g \cdot \mu)_{i j}=g_{i} \mu_{i j} g_{j}^{-1}
$$

We call this action the gauge action. The group $G$ contains the scalars, $\Delta: \mathbb{C}^{*} \rightarrow G$, $t \mapsto(t, \ldots, t)$, which act trivially. This leads us to also consider the quotient group $\widetilde{G}=G / \Delta$. 
The differential. Define $d: L^{n} \rightarrow L^{n+1}$ by the formula

$$
d \mu\left(a_{1}, \ldots, a_{n+1}\right)=\sum_{i=1}^{n}(-1)^{n-i} \mu\left(\ldots, a_{i} a_{i+1}, \ldots\right)
$$

For example, $d: L^{0} \rightarrow L^{1}$ is equal to zero, and $d: L^{1} \rightarrow L^{2}$ is given by $d \mu(a, b)=$ $\mu(a b)$.

Of course, $d^{2}=0$. The gauge action preserves the differential. The differential preserves the projective double grading. Note that the gauge group action on $L^{1}$ is not modified by a gauge term because $d: L^{0} \rightarrow L^{1}$ vanishes.

The complex $(L, d)$ is the subcomplex of internal degree zero of the Hochschild complex of the $\mathbb{C}$-algebra $\mathfrak{m}$ with values in the bimodule End $V$, where End $V$ has the trivial (i.e., zero) module structure.

The bracket. For $\mu \in L^{m}$ and $\mu^{\prime} \in L^{n}$ define $\mu \circ \mu^{\prime} \in L^{m+n}$ by the formula

$$
\mu \circ \mu^{\prime}\left(a_{1}, \ldots, a_{m+n}\right)=(-1)^{m n} \mu\left(a_{1}, \ldots, a_{m}\right) \circ \mu^{\prime}\left(a_{m+1}, \ldots, a_{m+n}\right) .
$$

An easy sign calculation shows that this operation is associative.

Then, for $\mu \in L^{m}$ and $\mu^{\prime} \in L^{n}$ define $\left[\mu, \mu^{\prime}\right] \in L^{m+n}$ by

$$
\left[\mu, \mu^{\prime}\right]=\mu \circ \mu^{\prime}-(-1)^{m n} \mu^{\prime} \circ \mu .
$$

This operation automatically satisfies the graded Jacobi identity because it is defined as the graded commutator of an associative product.

We can write out the formula for the bracket:

$$
\begin{array}{r}
{\left[\mu, \mu^{\prime}\right]\left(a_{1}, \ldots, a_{m+n}\right)=(-1)^{m n} \mu\left(a_{1}, \ldots, a_{m}\right) \circ \mu^{\prime}\left(a_{m+1}, \ldots, a_{m+n}\right)} \\
-\mu^{\prime}\left(a_{1}, \ldots, a_{n}\right) \circ \mu\left(a_{n+1}, \ldots, a_{m+n}\right) .
\end{array}
$$

For example, if $\mu, \mu^{\prime} \in L^{1}$, then

$$
\left[\mu, \mu^{\prime}\right](a, b)=-\mu(a) \circ \mu^{\prime}(b)-\mu^{\prime}(a) \circ \mu(b) .
$$

The differential $d$ acts as a derivation with respect to the bracket $[\cdot, \cdot]$, that is, for $\mu \in L^{m}$ and $\mu^{\prime} \in L^{n}$, we have

$$
d\left[\mu, \mu^{\prime}\right]=\left[d \mu, \mu^{\prime}\right]+(-1)^{m}\left[\mu, d \mu^{\prime}\right] .
$$

Thus $(L, d,[\cdot, \cdot])$ is a differential graded Lie algebra.

The gauge group $G$ acts by automorphisms of the differential graded Lie algebra structure on $L$. This means that we have

$$
d(g \cdot \mu)=g \cdot d \mu \quad \text { and } \quad g \cdot\left[\mu, \mu^{\prime}\right]=\left[g \cdot \mu, g \cdot \mu^{\prime}\right] .
$$

The derivative of the gauge action of $G$ on $L$ is the adjoint action of $L^{0}$ on $L$. 
Remark 1.1. The more basic object than $L$ is the truncation $L^{>0}=\tau_{>0} L$, together with $G$ and its gauge action. The differential graded Lie algebra $L$ can be recovered from $\left(L^{>0}, G\right)$.

The Maurer-Cartan equation. The Maurer-Cartan equation is

$$
d \mu+\frac{1}{2}[\mu, \mu]=0 \quad \text { for } \mu \in L^{1} .
$$

We call $\mu \in L^{1}$ a Maurer-Cartan element if it satisfies this equation. We denote the set of Maurer-Cartan elements by $M C(L)$.

For $\mu \in L^{1}$, we have $\frac{1}{2}[\mu, \mu]=\mu \circ \mu$, and so $\mu$ is a Maurer-Cartan element if and only if

$$
d \mu+\mu \circ \mu=0
$$

or, equivalently, if for all $a, b \in \mathfrak{m}$,

$$
\mu(a b)=\mu(a) \circ \mu(b) .
$$

If we write out this equation degreewise, we get for all $i>k>j, a \in \mathfrak{m}_{i-k}$ and $b \in \mathfrak{m}_{k-j}$, the equation $\mu_{i j}(a b)=\mu_{i k}(a) \circ \mu_{k j}(b)$.

Thus $\mu \in L^{1}$ is a Maurer-Cartan element if and only if it defines a left action of $\mathfrak{m}$ on $V$. Dividing by the gauge action removes the choice of basis in $V$. It follows immediately that Maurer-Cartan elements up to gauge equivalence are graded $\mathfrak{m}$-modules up to isomorphism, whose underlying graded vector space is isomorphic to $V$. We can make this claim precise:

Remark 1.2. Let $[M C(L) / G]$ be the (set-theoretic) transformation groupoid associated to the gauge group action on the Maurer-Cartan elements. Let (m-modules) $\alpha$ denote the category of graded $\mathfrak{m}$-modules with dimension vector $\alpha$ with only isomorphisms. Then we have an equivalence of groupoids

$$
[M C(L) / G] \longrightarrow(\mathfrak{m} \text {-modules })_{\alpha},
$$

given by mapping $\mu$ to the $m$-module structure it defines on $V$ and mapping an element of $G$ to the isomorphism of $\mathfrak{m}$-module structures it represents. We will turn this into a geometric statement.

1B. The moduli stack of $\boldsymbol{L}$. The following construction of the differential graded moduli stack works for any finite-dimensional differential graded Lie algebra concentrated in nonnegative degrees with algebraic gauge group.

Bundles of curved differential graded Lie algebras.

Definition 1.3. A bundle of curved differential graded Lie algebras over a scheme (or a stack) $M$ is a graded vector bundle $\mathscr{L}^{*}$ over $M$, endowed with three pieces of data: 
(i) a section $f \in \Gamma\left(M, \mathscr{L}^{2}\right)$,

(ii) an $\bigcirc_{M}$-linear map of degree one $\delta: \mathscr{L}^{*} \rightarrow \mathscr{L}^{*}$,

(iii) a $\mathscr{O}_{M}$-linear alternating bracket of degree zero $[\cdot, \cdot]: \Lambda^{2} \mathscr{L}^{*} \rightarrow \mathscr{L}^{*}$, subject to four axioms:

(i) $\delta(f)=0$, as a section of $\mathscr{L}^{3}$,

(ii) $\delta \circ \delta=[f, \cdot]$,

(iii) $\delta$ is a graded derivation with respect to the bracket $[\cdot, \cdot]$,

(iv) the bracket $[\cdot, \cdot]$ satisfies the graded Jacobi identity.

A bundle of curved differential graded Lie algebras is a bundle of differential graded Lie algebras only if $f=0$. All of our bundles of curved differential graded Lie algebras will be concentrated in degrees $\geq 2$. The section $f$ is the curving, and the map $\delta$ will be referred to as the twisted differential.

It will be useful to relax the conditions somewhat and call a sheaf of curved differential graded Lie algebras on $M$ a graded sheaf of $\mathcal{O}_{M}$-algebras, endowed with the same data (i) to (iii), subject to the same constraints (i) to (iv). Sheaves of curved differential graded Lie algebras will also be allowed to have contributions in degrees less than 2. The sheaf of Maurer-Cartan elements of a sheaf of curved differential graded Lie algebras is the preimage of $-f$ under the curvature map $\mathscr{L}^{1} \rightarrow \mathscr{L}^{2}$ given by $x \mapsto \delta x+\frac{1}{2}[x, x]$. If $\mathscr{L}$ is a bundle (so that $\mathscr{L}^{1}=0$ ), then the Maurer-Cartan locus is the scheme-theoretic vanishing locus of $f$ in $M$.

If $\mathscr{L}$ is a bundle of curved differential graded Lie algebras on $M$ and $\mathscr{R}_{M}$ a sheaf of differential graded $\mathscr{O}_{M}$-algebras, then $\mathscr{L}_{\mathscr{O}_{M}} \mathscr{R}_{M}$ is in a natural way a sheaf of curved differential graded Lie algebras.

We do not define the notion of morphism of bundles or sheaves of curved differential graded Lie algebras. More relevant is the notion of morphism of differential graded scheme, which, as we shall see, applies to bundles of curved differential graded Lie algebras.

Associated differential graded scheme or stack. To a bundle of curved differential graded Lie algebras over $M$ we associate a sheaf of differential graded algebras $\mathscr{R}_{M}$ by letting the underlying sheaf of graded $O_{M}$-algebras be

$$
\mathscr{R}_{M}^{*}=\operatorname{Sym}_{\bigcirc_{M}} \mathscr{L}[1]^{\vee},
$$

the sheaf of free graded commutative $\mathrm{O}_{M}$-algebras with unit on the (homologically) shifted dual of $\mathscr{L}$.

The bracket defines a morphism $q_{2}: \mathscr{L}[1]^{\vee} \rightarrow \operatorname{Sym}_{\mathscr{O}_{M}}^{2} \mathscr{L}[1]^{\vee}$, the twisted differential a morphism $q_{1}: \mathscr{L}[1]^{\vee} \rightarrow \operatorname{Sym}_{\mathscr{O}_{M}}^{1} \mathscr{L}[1]^{\vee}=\mathscr{L}[1]^{\vee}$ and the curving a morphism $q_{0}: \mathscr{L}[1]^{\vee} \rightarrow \operatorname{Sym}_{\mathscr{O}_{M}}^{0} \mathscr{L}[1]^{\vee}=\mathscr{O}_{M}$. All three morphisms $q_{i}$ have homological degree 
+1 , and all three extend uniquely to $\mathscr{O}_{M}$-linear derivations $q_{i}: \mathscr{R}_{M} \rightarrow \mathscr{R}_{M}$. Let $q=q_{0}+q_{1}+q_{2}$ be the sum of these three derivations. The four axioms of curved differential graded Lie algebra translate into the one condition

$$
q^{2}=0
$$

for the derivation $q$ on $\mathscr{R}_{M}$. This defines the differential graded scheme $\left(\mathscr{R}_{M}, q\right)$. We will usually suppress $q$ from the notation.

Note that $X=Z(f) \subset M$, the scheme theoretic vanishing locus of $f$ (the MaurerCartan locus), is equal to the subscheme of $M$ defined by the image of $\mathscr{R}^{-1}$ in $\mathscr{R}_{M}^{0}=\mathscr{O}_{M}$. The structure sheaf of $X$ is $O_{X}=h^{0}\left(\mathscr{R}_{M}\right)$.

Example 1.4. Given a finite-dimensional differential graded Lie algebra $L$, concentrated in degrees $>0$, we let $M=L^{1}=\operatorname{Spec} \operatorname{Sym}\left(L^{1 \vee}\right)$. Over $M$ we consider for every $i \geq 2$ the trivial vector bundle $\mathscr{L}^{i}$ with fiber $L^{i}$, that is, $\mathscr{L}^{i}=L^{i} \times M$. The curvature map $f: L^{1} \rightarrow L^{2}$ given by $f(x)=d x+\frac{1}{2}[x, x]$ gives rise to a section of $\mathscr{L}^{2}$ over $M$, the twisted differential $\delta=d^{\mu}: \mathscr{L}^{i} \rightarrow \mathscr{L}^{i+1}$ is defined by the formula $\delta(y)=d^{\mu}(y)=d y+[\mu, y]$ in the fiber over $\mu \in M=L^{1}$, and the bracket on $\mathscr{L}$ is constant, that is, equal to the bracket on $L$ in every fiber of $\mathscr{L}$. In this way the differential graded Lie algebra $L=L^{\geq 1}$ gives rise to a bundle of differential graded Lie algebras $\mathscr{L}=\mathscr{L}^{\geq 2}$ over $M=L^{1}$.

Note that $X=Z(f) \subset M$ is identified with the scheme theoretic Maurer-Cartan locus of $L$.

If an algebraic group $G$ acts on $L$ by automorphisms of the differential graded Lie algebra structure, the bundle of curved differential graded Lie algebras $\mathscr{L}$ over $M$ inherits a $G$-action covering the $G$-action on $M$ (this is just the diagonal action). Thus, the bundle of curved differential graded Lie algebras $\mathscr{L}$ descends to the quotient stack $[M / G]$.

We apply these considerations to the truncation of our differential graded Lie algebra $L^{>0}$ with the gauge group action by $G$. We obtain a bundle of curved differential graded Lie algebras $\mathscr{L}_{\mathfrak{M}}$ over $\mathfrak{M}=[M / G]$ and a sheaf of differential graded algebras $\mathscr{R}_{\mathfrak{M}}$ over $\mathfrak{M}$.

If we replace $G$ by $\widetilde{G}$, we obtain a bundle of curved differential graded Lie algebras $\mathscr{L}_{\mathfrak{M}}$ over $\tilde{\mathfrak{M}}=[M / \widetilde{G}]$ and a sheaf of differential graded algebras $\mathscr{R}_{\mathfrak{M}_{\mathfrak{M}}}$ over $\widetilde{\mathfrak{M}}$. The Maurer-Cartan locus $X=Z(f) \subset M$ descends to closed substacks $\mathfrak{X} \subset \mathfrak{M}$ and $\widetilde{\mathfrak{X}} \subset \widetilde{\mathfrak{M}}$ such that $\mathcal{O}_{\mathfrak{X}}=h^{0}\left(\mathscr{R}_{\mathfrak{M}}\right)$ and $\mathscr{O}_{\mathfrak{X}}=h^{0}\left(\mathscr{R}_{\mathfrak{M}}\right)$.

Remark 1.5. There is a natural morphism $\mathfrak{M} \rightarrow \tilde{\mathfrak{M}}$, making $\mathfrak{M}$ a $\mathbb{C}^{*}$-gerbe over $\widetilde{\mathfrak{M}}$. This gerbe is trivial if there exists a line bundle $\xi$ over $M$ and a lifting of the $G$-action to a $G$-action on $\xi$ such that $\Delta$ acts by scalar multiplication on the fibers of $\xi$. 
The associated functor on differential graded schemes. Suppose the differential graded scheme $\left(M, \mathscr{R}_{M}\right)$ comes from a bundle of curved differential graded Lie algebras as in Equation (4). Given a morphism of schemes $\phi: T \rightarrow M$, the sheaf of Maurer-Cartan elements of $\phi^{*} \mathscr{L} \otimes_{\bigcirc_{T}} \mathscr{R}_{T}$ is naturally isomorphic to the sheaf of morphisms of differential graded $\mathscr{O}_{T}$-algebras $\phi^{*} \mathscr{R}_{M} \rightarrow \mathscr{R}_{T}$.

$$
M C\left(\phi^{*} \mathscr{L} \otimes_{\mathfrak{O}_{T}} \mathscr{R}_{T}\right)=\operatorname{Mor}_{\mathscr{C}_{T}}\left(\phi^{*} \operatorname{Sym}_{\mathscr{O}_{M}} \mathscr{L}[1]^{\vee}, \mathscr{R}_{T}\right) .
$$

In particular, a morphism of differential graded schemes $\left(T, \mathscr{R}_{T}\right) \rightarrow\left(M, \mathscr{R}_{M}\right)$ is essentially the same thing as a pair $(\phi, \mu)$, where $\phi: T \rightarrow M$ is a morphism of schemes and $\mu$ is a global Maurer-Cartan element of the sheaf of curved differential graded Lie algebras $\phi^{*} \mathscr{L} \otimes_{\bigcirc_{T}} \mathscr{R}_{T}$.

Lemma 1.6. If $\left(M, \mathscr{R}_{M}\right)$ comes as in Example 1.4 from a differential graded Lie algebra $L=L^{\geq 1}$, then a morphism $\left(T, \mathscr{R}_{T}\right) \rightarrow\left(M, \mathscr{R}_{M}\right)$ is the same thing as a global Maurer-Cartan element in the sheaf of differential graded Lie algebras $L \otimes_{\mathbb{C}} \mathscr{R}_{T}$.

Proof. Start with a morphism of differential graded schemes

$$
(\phi, \mu):\left(T, \mathscr{R}_{T}\right) \rightarrow\left(M, \mathscr{R}_{M}\right),
$$

where we think of $\mu$ as a global Maurer-Cartan element in the sheaf of curved differential graded Lie algebras $\phi^{*} \mathscr{L} \otimes_{\mathcal{O}_{Y}} \mathscr{R}_{T}$. The underlying morphism of schemes $\phi: T \rightarrow L^{1}$ can be considered as a section of $L^{1} \otimes \mathscr{L}^{0}\left(\mathscr{R}_{T}\right)$ over $T$ and hence as a degree 1 section of $L \otimes \mathscr{R}_{T}$. The section $\mu$ can also be thought of as a degree 1 section $L \otimes \mathscr{R}_{T}$, and it is not hard to check that $\mu+\phi$ is a Maurer-Cartan section of the sheaf of differential graded Lie algebras $L \otimes \mathscr{R}_{T}$. Conversely, every MaurerCartan section of $L \otimes \mathscr{R}_{T}$ gives rise to a pair $(\phi, \mu)$ and hence to a morphism of differential graded schemes $\left(T, \mathscr{R}_{T}\right) \rightarrow\left(M, \mathscr{R}_{M}\right)$.

Finally, if $G$ acts on $L$ by automorphisms and $\mathfrak{M}=[M / G]$, then a morphism $\left(T, \mathscr{R}_{T}\right) \rightarrow\left(\mathfrak{M}, \mathscr{R}_{\mathfrak{M}}\right)$ is essentially the same thing as a pair $(E, \mu)$, where $E$ is a principal $G$-bundle over $T$, and $\mu$ is a global Maurer-Cartan element of the sheaf of differential graded Lie algebras ${ }^{E} L \otimes_{\mathcal{O}_{T}} \mathscr{R}_{T}$. Here ${ }^{E} L$ denotes the associated vector bundle with its induced structure of sheaf of differential graded Lie algebras over $\mathrm{O}_{T}$.

The derived scheme of actions. We apply these considerations to the differential graded Lie algebra

$$
L^{\geq 1}=\operatorname{Hom}_{\mathrm{gr}}\left(\mathfrak{m}^{\otimes \geq 1}, \text { End } V\right) .
$$

Let $\left(M, \mathscr{R}_{M}\right)$ be the differential graded scheme associated as in Example 1.4 to $L^{\geq 1}=\operatorname{Hom}_{\mathrm{gr}}\left(\mathfrak{m}^{\otimes \geq 1}\right.$, End $\left.V\right)$. So $M=\operatorname{Hom}_{\mathrm{gr}}(\mathfrak{m}$, End $V)$. The following is 
essentially Proposition (3.5.2) of [Ciocan-Fontanine and Kapranov 2001]. (See the same work for the definition of $A_{\infty}$-action.)

Proposition 1.7. Suppose $\left(T, \mathscr{R}_{T}\right)$ is a differential graded scheme. A morphism $\left(T, \mathscr{R}_{T}\right) \rightarrow\left(M, \mathscr{R}_{M}\right)$ is the same thing as a Maurer-Cartan element in the differential graded Lie algebra

$$
\Gamma\left(T, \operatorname{Hom}\left(\mathfrak{m}^{\otimes \geq 1}, \text { End } V\right) \otimes \mathscr{R}_{T}\right)
$$

This, in turn, is the same thing as a graded $\mathscr{R}_{T}$-linear $A_{\infty}$-action of $\mathfrak{m} \otimes \mathscr{R}_{T}$ on $V \otimes \mathscr{R}_{T}$ or a graded unital $\mathscr{R}_{T}$-linear $A_{\infty}$-action of $A \otimes \mathscr{R}_{T}$ on $V \otimes \mathscr{R}_{T}$.

This justifies calling $\left(M, \mathscr{R}_{M}\right)$ the derived scheme of graded actions of $A$ on $V$ and denoting it by $\Re \operatorname{Act}_{\mathrm{gr}}(A, V)$.

The derived stack of modules. Let $\left(\mathfrak{M}, \mathscr{R}_{\mathfrak{M}}\right)$ be the differential graded stack obtained from $\left(M, \mathscr{R}_{M}\right)$ by dividing by $G$, and let $\mathfrak{X} \subset \mathfrak{M}$ be the Maurer-Cartan locus.

Proposition 1.8. Suppose $\left(T, \mathscr{R}_{T}\right)$ is a differential graded scheme. A morphism $\left(T, \mathscr{R}_{T}\right) \rightarrow\left(\mathfrak{M}, \mathscr{R}_{\mathfrak{M}}\right)$ is the same thing as a pair $(E, \mu)$, where $E=\bigoplus_{i=p}^{q} E_{i}$ is a graded vector bundle of dimension vector $\alpha$ over $T$, and $\mu$ is a Maurer-Cartan element in the differential graded Lie algebra

$$
\Gamma\left(T, \mathscr{H o m}_{\mathrm{gr}}\left(\mathfrak{m}^{\otimes \geq 1}, \mathscr{E} \mathrm{d}_{\mathbb{O}_{T}} E\right) \otimes_{\mathcal{O}_{T}} \mathscr{R}_{T}\right)
$$

Such a Maurer-Cartan element $\mu$ is the same thing as a graded $\mathscr{R}_{T}$-linear $A_{\infty}$ action of $\mathfrak{m} \otimes \mathscr{R}_{T}$ on $E \otimes_{\mathcal{O}_{T}} \mathscr{R}_{T}$, or a graded unital $\mathscr{R}_{T}$-linear $A_{\infty}$-action of $A \otimes \mathscr{R}_{T}$ on $E \otimes_{O_{T}} \mathscr{R}_{T}$.

In particular, if $T$ is a classical scheme, a morphism $T \rightarrow\left(\mathfrak{M}, \mathscr{R}_{\mathfrak{M}}\right)$ is the same thing as a morphism $T \rightarrow \mathfrak{X}$, which, in turn, is the same thing as a graded vector bundle over $T$ of dimension $\alpha$, endowed with the structure of a sheaf of graded $\mathfrak{m} \otimes \mathrm{O}_{T}$-modules or the structure of a sheaf of graded unital $A \otimes \mathrm{O}_{T}$-modules.

There is a universal family over $\left(\mathfrak{M}, \mathscr{R}_{\mathfrak{M}}\right)$. It is obtained from $V \otimes \mathscr{O}_{M}$ with its tautological $A_{\infty}$-action

$$
\boldsymbol{\mu}: \mathfrak{m} \otimes V \otimes \mathscr{R}_{M} \longrightarrow V \otimes \mathscr{R}_{M}
$$

by descent: the group $G$ acts naturally on $V$ in a way respecting $\boldsymbol{\mu}$.

We call $\left(\mathfrak{M}, \mathscr{R}_{\mathfrak{M}}\right)$ the derived stack of graded $A$-modules with dimension vector $\alpha$, and use the notation $\mathfrak{R M o d}_{\alpha}(A)=\left(\mathfrak{M}, \mathscr{R}_{\mathfrak{M}}\right)$. For the underlying classical stack $\mathfrak{X}$, we write $\mathfrak{M o d}_{\alpha}(A)=\mathfrak{X}$. 
1C. The derived space of equivalence classes of simple modules. When dividing by $\widetilde{G}$ instead of $G$, we have to be more careful because the natural action of $G$ on $V$ does not factor through $\widetilde{G}$ as the scalars in $G$ do not act trivially on $V$. This implies that the universal family of graded $A$-modules does not descend from $\mathfrak{M}$ to $\tilde{\mathfrak{M}}$. The obstruction is the $\mathbb{C}^{*}$-gerbe of Remark 1.5.

Equivalence of simple modules. A family of graded A-modules of dimension $\alpha$ parametrized by the scheme $T$ is a graded vector bundle with rank vector $\alpha$ on $T$ together with a unital graded $O_{T}$-linear action of $A \otimes O_{T}$.

Definition 1.9. A family $E$ of graded $A$-modules parametrized by $T$ is simple if the sheaf of endomorphisms of $E$ is equal to $\mathcal{O}_{T}$. Two simple families of graded $A$-modules $E, F$, parametrized by $T$ are equivalent, if there exists a line bundle $\mathscr{L}$ on $T$, such that $F$ is isomorphic to $E \otimes_{\bigcirc_{T}} \mathscr{L}$, as a family of graded $A$-modules.

Equivalence classes of simple families of graded $A$-modules form a presheaf on the site of $\mathbb{C}$-schemes with the étale topology, whose associated sheaf we denote by $\tilde{\mathfrak{M}}_{\alpha}^{\mathrm{sp}}(A)$.

Let $M^{\text {sp }} \subset M$ be the open subscheme of points with trivial $\widetilde{G}$-stabilizer, and $X^{\text {sp }}=X \cap M$ the intersection with the Maurer-Cartan locus $X$. Denote by $\tilde{\mathfrak{M}}^{\text {sp }} \subset \widetilde{\mathfrak{M}}$ and $\widetilde{\mathfrak{X}}^{\text {sp }} \subset \widetilde{\mathfrak{X}}$ the quotients by $\widetilde{G}$.

Remark 1.10. The sheaf $\tilde{\mathfrak{M}}_{o d_{\alpha}}^{\mathrm{sp}}(A)$ is isomorphic to the algebraic space $\tilde{\mathfrak{X}}^{\mathrm{sp}}$.

$$
\tilde{\mathfrak{M}}_{\alpha}^{\mathrm{sp}}(A)=\tilde{\mathfrak{X}}^{\mathrm{sp}}
$$

This proves that $\tilde{\mathfrak{M}}_{\alpha}^{\mathrm{sp}}(A)$ is algebraic, and gives a modular interpretation of $\tilde{\mathfrak{X}}^{\mathrm{sp}}$. Coprime case.

Proposition 1.11. Suppose that the components of the dimension vector $\alpha$ are coprime. Then the gerbe of Remark 1.5 is trivial. Moreover, the presheaf of equivalence classes of simple families of graded A-modules is a sheaf. In other words, for any $\mathbb{C}$-scheme $T$, the $T$-points of the algebraic space $\tilde{\mathfrak{M}}_{\alpha}^{\mathrm{sp}}(A)$ correspond one-to-one to equivalence classes of simple families. In particular, $\tilde{\mathfrak{M}}_{\mathrm{od}}^{\mathrm{sp}}(A)$ admits a universal family of simple graded A-modules.

Proof. There exist integers $n_{i}$ such that $\sum_{i=p}^{q} n_{i} \alpha_{i}=1$. The character $\rho: G \rightarrow \mathbb{C}^{*}$ given by $\rho(g)=\prod_{i=p}^{q} \operatorname{det}\left(g_{i}\right)^{n_{i}}$ satisfies $\langle\Delta, \rho\rangle=1$. So twisting the action of $G$ on $V$ by $\rho^{-1}$, the twisted action factors through $\widetilde{G}$, and so after the twist, $V$ descends to $\tilde{\mathfrak{M}}$.

Remark 1.12. If

$$
\alpha(t)=a_{0}\left(\begin{array}{l}
t \\
0
\end{array}\right)+a_{1}\left(\begin{array}{l}
t \\
1
\end{array}\right)+\cdots+a_{k}\left(\begin{array}{l}
t \\
k
\end{array}\right)
$$


is a numerical polynomial $\alpha(t) \in \mathbb{Q}[t]$ of degree $k$ with $a_{0}, \ldots, a_{k} \in \mathbb{Z}$, and $q-p \geq k$, then

$$
(\alpha(p), \ldots, \alpha(q))=1 \Longleftrightarrow\left(a_{0}, \ldots, a_{k}\right)=1 .
$$

Hence $(\alpha(p), \ldots, \alpha(q))=1$ if and only if $\alpha$ is primitive (not an integer multiple of another numerical polynomial).

We will write down the derived moduli problem solved by the differential graded algebraic space $\left(\tilde{\mathfrak{M}}^{\mathrm{sp}}, \mathscr{R}\right)$.

The derived space of simple modules. Let $\left(T, \mathscr{R}_{T}\right)$ be a differential graded scheme. If $F$ is a graded vector bundle on $T$, we can sheafify the construction of our differential graded Lie algebra over $T$, and tensor with $\mathscr{R}_{T}$ to obtain a sheaf of differential graded Lie algebras

$$
\mathscr{H}_{\mathrm{gr}}\left(\mathfrak{m}^{\otimes \geq 1}, \mathscr{E} n d_{\mathscr{O}_{T}} F\right) \otimes_{\mathscr{O}_{T}} \mathscr{R}_{T} .
$$

A global Maurer-Cartan element in (5) is the same thing as a graded $\mathscr{R}_{T}$-linear $A_{\infty}$-action of $\mathfrak{m} \otimes \mathscr{R}_{T}$ on $F \otimes_{\mathcal{O}_{T}} \mathscr{R}_{T}$.

A family of graded A-modules with dimension vector $\alpha$ parametrized by the differential graded scheme $\left(T, \mathscr{R}_{T}\right)$ is a pair $(F, \mu)$, where $F$ is a graded vector bundle of dimension $\alpha$ over $T$, and $\mu$ is a global Maurer-Cartan element in (5). Two such families are equivalent if they differ by a line bundle on $T$. We denote the set of equivalence classes of such families by $\mathfrak{R} \tilde{\mathfrak{M}}_{\alpha}(A)(T)$. Varying $\left(T, \mathscr{R}_{T}\right)$, we get a presheaf $\mathfrak{R} \tilde{\mathfrak{M}}_{\alpha}(A)$ on the category of differential graded schemes.

Note that a Maurer-Cartan element $\mu$ in (5) can be decomposed

$$
\mu=\sum_{i=1}^{q-p} \mu_{i}, \quad \mu_{i} \in \mathscr{H} \operatorname{lom}_{\mathrm{gr}}\left(\mathfrak{m}^{\otimes i}, \mathscr{\complement} n d_{\bigcirc_{T}} F\right) \otimes_{\bigcirc_{T}} \mathscr{R}_{T}^{1-i}
$$

So $\mu_{1} \in \mathscr{H}_{\text {om }}\left(\mathfrak{m}, \stackrel{\mathscr{E}}{E} d_{\mathbb{O}_{T}} F\right) \otimes_{\mathscr{O}_{T}} \mathscr{R}_{T}^{0}$. The Maurer-Cartan equation implies that $\mu_{1}$ takes values in the subsheaf $\mathscr{H} \operatorname{lom}_{\mathrm{gr}}\left(\mathfrak{m}, \mathscr{E} n d_{\bigcirc_{T}} F\right) \otimes_{\mathscr{O}_{T}} \mathscr{E}^{0}\left(\mathscr{R}_{T}\right)$, which is equal to $\mathscr{H} \operatorname{lom}_{\mathrm{gr}}\left(\mathfrak{m}, \mathscr{E} n d_{\mathscr{C}_{T}} F\right)$ by our definition of differential graded scheme. Thus, we may also think of $\mu_{1}$ as an $\mathscr{O}_{T}$-linear map $\mu_{1}: \mathfrak{m} \otimes \mathscr{O}_{T} \rightarrow \mathscr{E} n d_{\mathscr{O}_{T}} F$. We call $(F, \mu)$ simple if the subsheaf of $\mathscr{E} n d_{\mathscr{O}_{T}} F$ commuting with the image of $\mu_{1}$ is equal to $\mathscr{O}_{T}$. Simple families define the subpresheaf $\mathfrak{R} \tilde{M}_{0}^{\text {sp }}(A) \subset \mathfrak{R} \widetilde{\mathfrak{M}}_{\alpha}(A)$.

Proposition 1.13. The differential graded algebraic space $\left(\tilde{\mathfrak{M}}^{\mathrm{sp}}, \mathscr{R}\right)$ represents the

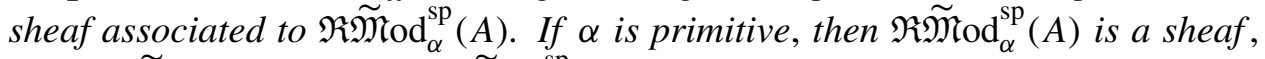
and so $\left(\tilde{\mathfrak{M}}^{\mathrm{sp}}, \mathscr{R}\right)$ represents $\mathfrak{R} \tilde{\mathrm{M}}_{\mathrm{Tod}}^{\mathrm{sp}}(A)$.

Proof. Let $(F, \mu)$ be a simple graded family parametrized by the differential graded scheme $\left(T, \mathscr{R}_{T}\right)$. Write $\mu=\mu_{1}+\mu^{\prime}$, where $\mu^{\prime}=\sum_{i \geq 2} \mu_{i}$. Then the pair $\left(F, \mu_{1}\right)$ defines a morphism $\phi: T \rightarrow \widetilde{\mathfrak{M}}^{\mathrm{sp}}$, and any equivalent simple graded family gives 
rise to the same morphism $T \rightarrow \widetilde{\mathfrak{M}}^{\text {sp }}$. The pullback to $T$ of $\mathscr{R}_{\mathfrak{M}}$ via the morphism $\phi$ is equal to the sheaf of symmetric algebras generated over $O_{T}$ by the shifted dual of $\mathscr{H}_{\mathrm{om}}\left(\mathfrak{m} \geq 2, \mathscr{E} n d_{\mathscr{O}_{T}} F\right)$. Therefore, a morphism $\phi^{*} \mathscr{R}_{\tilde{M}_{\mathfrak{M}}} \rightarrow \mathscr{R}_{T}$ is the same thing as a global Maurer-Cartan section of the sheaf of curved differential graded Lie algebras (with twisted differential)

$$
\mathscr{H o m}_{\mathrm{gr}}\left(\mathfrak{m}^{\geq 2}, \mathscr{E} n d_{\mathscr{O}_{T}} F\right) \otimes_{\mathscr{O}_{T}} \mathscr{R}_{T} .
$$

This is exactly what $\mu^{\prime}$ provides us with. Hence $(F, \mu)$ gives rise to a morphism $\left(T, \mathscr{R}_{T}\right) \rightarrow(\widetilde{\mathfrak{M}}, \mathscr{R})$.

We have defined a morphism from the presheaf $\mathfrak{R} \tilde{\mathfrak{M}}_{\alpha}^{\mathrm{sp}}(A)$ to the sheaf represented by $\left(\tilde{\mathfrak{M}}^{\mathrm{sp}}, \mathscr{R}\right)$. Conversely, every morphism $\phi: T \rightarrow \widetilde{\mathfrak{M}}^{\mathrm{sp}}$ is (locally in $T$ )

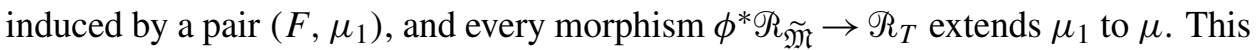
proves that every section of $\left(\tilde{\mathfrak{M}}^{\mathrm{sp}}, \mathscr{R}\right)$ comes locally from a section of $\mathfrak{R} \tilde{\mathfrak{M}}_{\alpha}^{\mathrm{sp}}(A)$. This finishes the proof.

1D. The tangent complex. Suppose $\mathscr{L}=\mathscr{L}^{\geq 2}$ is a bundle of curved differential graded Lie algebras on the smooth scheme (or algebraic space) $M$, and let $X \subset M$ be its Maurer-Cartan locus. As a direct consequence of the second axiom (Definition 1.3), the restriction of $(\mathscr{L}, \delta)$ to $X$ is a complex of sheaves of $O_{X^{-}}$ modules. The derivative of the curving $f: M \rightarrow \mathscr{L}^{2}$ gives rise to an $\mathbb{O}_{X}$-linear map $\left.\left.T_{M}\right|_{X} \rightarrow \mathscr{L}^{2}\right|_{X}$, and we obtain an augmented complex

$$
\Theta^{\bullet}=\left[\left.\left.\left.T_{M}\right|_{X} \longrightarrow \mathscr{L}^{2}[1]\right|_{X} \longrightarrow \mathscr{L}^{3}[1]\right|_{X} \longrightarrow \cdots\right]
$$

by the first axiom. This complex $\Theta^{\bullet}$ of vector bundles on $X$ is called the tangent complex of $(M, \mathscr{L})$. The shifts are applied to $\left.\mathscr{L}\right|_{X}$ so that $\left.T_{M}\right|_{X}$ will end up in degree 0 .

By construction, the 0 -th cohomology sheaf is equal to the Zariski tangent sheaf of $X$ :

$$
h^{0}\left(\Theta^{\bullet}\right)=T_{X}
$$

Next, we will recall how $\Theta$ governs deformation and obstruction theory.

Deformation theory for small extensions. Consider a pointed differential graded algebra $A \rightarrow \mathbb{C}$, concentrated in nonpositive degrees. Let $A^{\prime} \rightarrow A$ be a small extension of differential graded algebras: this means that the kernel $I$ defined by the short exact sequence

$$
0 \longrightarrow I \longrightarrow A^{\prime} \longrightarrow A \longrightarrow 0,
$$

and the kernel of the augmentation $A^{\prime} \rightarrow \mathbb{C}$ annihilate each other. This implies that the $A^{\prime}$-module structure on $I$ is induced from the $\mathbb{C}$-vector space structure on $I$ via the augmentation $A^{\prime} \rightarrow \mathbb{C}$. For simplicity, assume that $I$ is concentrated in a 
specific degree $-r \leq 0$. (The classical case is the case $r=0$.) Denote by $\left(T, \mathscr{R}_{T}\right)$ and $\left(T^{\prime}, \mathscr{R}_{T^{\prime}}\right)$ the affine differential graded schemes associated to $A$ and $A^{\prime}$.

We will consider a diagram

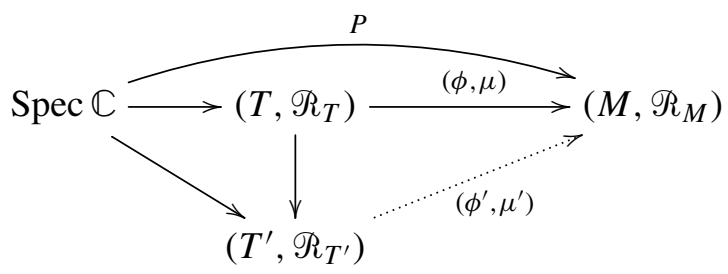

and ask for an obstruction to the existence of the dotted arrow. If a dotted arrow exists, we will classify all possible dotted arrows up to homotopy equivalence (see, for example, [Ciocan-Fontanine and Kapranov 2001] or [Manetti 1999] for the definition of homotopy equivalence).

Proposition 1.14. There exists a naturally defined element $h \in H^{1}\left(P^{*} \Theta \otimes I\right)$, which vanishes if and only if a dotted arrow exists in (6). If $h=0$, then the set of all dotted arrows in (6), up to homotopy equivalence, is in a natural way a principal homogeneous space for the abelian group $H^{0}\left(P^{*} \Theta \otimes I\right)$.

Proof. The morphism $(\phi, \mu):\left(T, \mathscr{R}_{T}\right) \rightarrow\left(M, \mathscr{R}_{M}\right)$ is given by a morphism of schemes $\phi: T \rightarrow M$ and a Maurer-Cartan element $\mu \in M C\left(\phi^{*} \mathscr{L} \otimes_{A^{0}} A\right)$. As $M$ is smooth, there is no obstruction to the existence of $\phi^{\prime}$, so let us choose $\phi^{\prime}$. Now consider the square zero extension of curved differential graded Lie algebras

$$
0 \longrightarrow P^{*} \mathscr{L} \otimes I \longrightarrow \phi^{\prime *} \mathscr{L} \otimes_{A^{\prime 0}} A^{\prime} \longrightarrow \phi^{*} \mathscr{L} \otimes_{A^{0}} A \longrightarrow 0 .
$$

We have a Maurer-Cartan element $\mu$ in the curved differential graded Lie algebra on the right, which means that

$$
f-\delta \mu+\frac{1}{2}[\mu, \mu]=0 .
$$

We lift $\mu$ at random to an element $\mu^{\prime}$ of the curved differential graded Lie algebra in the middle. The obstruction $h$ is defined as

$$
h=f^{\prime}-\delta \mu^{\prime}+\frac{1}{2}\left[\mu^{\prime}, \mu^{\prime}\right],
$$

which is an element of $P^{*} \mathscr{L} \otimes I$ and moreover a 2-cocycle in $P^{*} \mathscr{L} \otimes I$, hence a 1-cocycle in $P^{*} \Theta \otimes I$. The proof that the vanishing of $h$ in cohomology is equivalent to the existence of the dotted arrow distinguishes between the cases that $r=0$ and $r<0$. For $r<0$, we have $H^{2}\left(P^{*} \mathscr{L} \otimes I\right)=H^{1}\left(P^{*} \Theta \otimes I\right)$, and changing $\phi^{\prime}$ while fixing $\phi$ is impossible. So the question is if there exists $z \in P^{*} \mathscr{L} \otimes I$ of degree 1, such that $\mu^{\prime}+z$ is a Maurer-Cartan element in the middle of (7). Such a $z$ will exhibit $h$ as a coboundary (and conversely). For $r=0$, the element 
$h \in H^{1}\left(P^{*} \Theta \otimes I\right)$ is the classical obstruction to the existence of the dotted arrow in the diagram of classical schemes

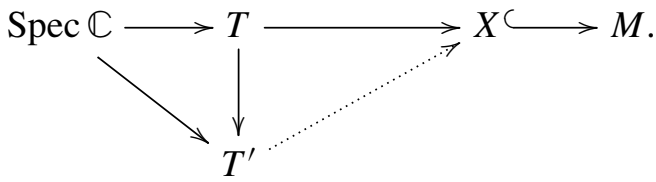

Now assume that the obstruction vanishes. The difference between any two Maurer-Cartan lifts of $\mu$ defines an element of $H^{0}\left(P^{*} \Theta \otimes I\right)$. One checks that this difference is a coboundary if and only if the two lifts define homotopy equivalent dotted arrows.

Corollary 1.15. For example, if $I=\mathbb{C}[r]$, then the obstructions are contained in $H^{r+1}\left(P^{*} \Theta\right)$ and the deformations are classified by $H^{r}\left(P^{*} \Theta\right)$.

Deformations of modules. Let us examine the meaning of Proposition 1.14 for the differential graded algebraic space $\left(\tilde{\mathfrak{M}}^{\mathrm{sp}}, \mathscr{R}\right)=\mathfrak{R} \tilde{\mathfrak{M}}_{\alpha}^{\mathrm{sp}}(A)$.

So let the $\mathbb{C}$-valued point $P: \operatorname{Spec} \mathbb{C} \rightarrow\left(\widetilde{\mathfrak{M}}^{\mathrm{sp}}, \mathscr{R}\right)$ be represented by the MaurerCartan element $\mu \in L$.

Lemma 1.16. The complex $\left(L, d^{\mu}\right)$ is precisely the graded normalized Hochschild cochain complex with coefficients in (End $V, \mu$ ), that is, End $V$ endowed with the structure of an A-A-bimodule from $\mu$.

Proof. This is immediate. The normalized or reduced complex is defined, for example, in [Loday 1992, §1.5.7].

Corollary 1.17. The complex $P^{*} \Theta$ is quasiisomorphic to the augmented graded Hochschild complex

$$
\mathbb{C} \longrightarrow \operatorname{End}_{\mathrm{gr}} V \longrightarrow \operatorname{Hom}_{\mathrm{gr}}(A, \text { End } V) \longrightarrow \operatorname{Hom}_{\mathrm{gr}}\left(A^{\otimes 2}, \text { End } V\right) \longrightarrow \cdots
$$

Proof. This follows immediately from the fact that the normalized Hochschild complex is quasiisomorphic to the Hochschild complex, by [Loday 1992, §1.5.7].

Corollary 1.18. Suppose that $P$ corresponds to the A-module $E=(V, \mu)$. Then we have

$$
H^{i}\left(P^{*} \Theta\right)= \begin{cases}\operatorname{Ext}_{A}^{i}(E, E)_{\mathrm{gr}} & \text { if } i>0, \\ \operatorname{Hom}_{A}(E, E)_{\mathrm{gr}} / \mathbb{C} & \text { for } i=0 .\end{cases}
$$

The tangent complex $\Theta$ itself is quasiisomorphic to the augmented complex

$$
\mathbb{C} \longrightarrow R \mathscr{H}^{\circ}{ }_{A}(\mathscr{E}, \mathscr{E}) \mathrm{gr},
$$

where $\mathscr{E}$ is the universal family of graded A-modules on $\widetilde{\mathfrak{X}}^{\mathrm{sp}}=\tilde{\mathfrak{M}}_{\text {od }}^{\mathrm{sp}}(A)$. 
Proof. This is a consequence of the standard fact that (graded) Hochschild cohomology computes (graded) extension spaces. A proof in the ungraded case can be found in Lemma 1.9.1 of [Weibel 1994].

Corollary 1.19. In a situation given by a diagram such as (6), assume that $I=\mathbb{C}[r]$, as in Corollary 1.15. Then obstructions are contained in $\operatorname{Ext}_{A}^{r+1}(E, E)_{\mathrm{gr}}$ and deformations are classified by $\operatorname{Ext}_{A}^{r}(E, E)_{\mathrm{gr}}\left(\right.$ or $\operatorname{Hom}_{A}(E, E)_{\mathrm{gr}} / \mathbb{C}$, for $\left.r=0\right)$.

\section{Stability}

We will apply geometric invariant theory to the construction of the quotient of $M=L^{1}$ by the gauge group $G$ as a quasiprojective scheme.

First, since the scalars in $G$ act trivially, no point of $L^{1}$ can be stable for the action of $G$. This prompts us to replace $G$ by $\widetilde{G}=G / \Delta$. Second, the canonical oneparameter subgroup $\lambda_{0}(t)=\left(t^{p}, \ldots, t^{q}\right)$ is central and acts by (see Equation (3))

$$
\left(\lambda_{0}(t) \cdot \mu\right)_{i j}=t^{i-j} \mu_{i j}
$$

and hence destabilizes every element of $L^{1}$, as $i>j$ if $\mu_{i j} \neq 0$. Thus the affine quotient $\operatorname{Spec} \mathbb{C}\left[L^{1}\right]^{G}$ is trivial, equal to $\operatorname{Spec} \mathbb{C}$.

In fact, the quotient of $L^{1}$ by $G$ classifies quiver representations for a certain quiver, and so we are in the situation worked out by King [1994]. Our quiver has $q-p+1$ vertices labeled $p, \ldots, q$, and for every pair of vertices $i<j$, there are $\operatorname{dim} A_{j-i}$ arrows from $i$ to $j$. The vector space $L^{1}=\operatorname{Hom}_{\mathrm{gr}}(\mathfrak{m}$, End $V)$ is denoted $\mathscr{R}(Q, \alpha)$ by King; the group $G$ is denoted by $\operatorname{GL}(\alpha)$.

To linearize the action of $\widetilde{G}$ on $L^{1}$, we choose a vector of integers

$$
\theta=\left(\theta_{p}, \ldots, \theta_{q}\right) \quad \text { such that } \sum_{i=p}^{q} \theta_{i} \alpha_{i}=0 .
$$

This defines the character $\chi_{\theta}: \widetilde{G} \rightarrow \mathbb{C}$ by

$$
\chi_{\theta}(g)=\prod_{i=p}^{q} \operatorname{det}\left(g_{i}\right)^{\theta_{i}},
$$

which we use to linearize the action.

For a graded vector subspace $W \subset V$, define

$$
\theta(W)=\sum_{i=p}^{q} \theta_{i} \operatorname{dim} W_{i}
$$

Note that whether or not $\mu \in L^{1}$ satisfies the Maurer-Cartan equation, it makes sense to speak of graded submodules $W \subset V$ with respect to $\mu$. 
Proposition 2.1 (King). The point $\mu \in L^{1}$ is (semi)stable for the action of $\widetilde{G}$ linearized by $\chi_{\theta}$ if and only if for every proper graded $\mu$-submodule $0<W<V$ we have $\theta(W)(\geq) 0$. (Here we use the usual convention that to characterize stability, the strict inequality applies, and for semistability the weak inequality is used.)

Denote by $L^{s}$ and $L^{s s}$ the open subsets of $L^{1}$ of stable and semistable points, respectively. Similarly, denote by $X^{s}$ and $X^{s s}$ the open subsets of stable and semistable points inside the Maurer-Cartan subscheme $X \subset L^{1}$.

The geometric invariant theory quotient of $L^{1}$ by $\widetilde{G}$ is the projective scheme

$$
L^{1} / / \widetilde{G}=\operatorname{Proj} \bigoplus_{n=0}^{\infty} \mathbb{C}\left[L^{1}\right]^{G, \chi^{n}}
$$

where $\mathbb{C}\left[L^{1}\right]^{G, \chi^{n}}=\left\{f: L^{1} \rightarrow \mathbb{C} \mid f(g x)=\chi^{n}(g) f(x)\right\}$ is the space of $\chi^{n}$-twisted invariants of $G$ in $\mathbb{C}\left[L^{1}\right]$. The quotient $L^{1} / / \widetilde{G}$ is indeed projective since $\mathbb{C}\left[L^{1}\right]^{G}=\mathbb{C}$.

Corollary 2.2. The scheme $L^{s} / / \widetilde{G}$ is a quasiprojective smooth scheme contained as an open subscheme in the algebraic space $\tilde{\mathfrak{M}}^{\mathrm{sp}}$. It is a locally fine moduli space for equivalence classes of stable quiver representations. In the coprime case, it is a fine moduli space.

The scheme $L^{1} / / \widetilde{G}=L^{s s} / / \widetilde{G}$ is a projective scheme containing $L^{s} / / \widetilde{G}$ as an open subscheme. Its points are in one-to-one correspondence with $S$-equivalence classes of semistable quiver representations.

Corollary 2.3. The differential graded scheme $\left(L^{s} / / \widetilde{G}, \mathscr{R}\right)$ is a quasiprojective differential graded scheme, which represents the sheaf associated to $\mathfrak{R} \widetilde{\mathfrak{M}}_{\alpha}^{s}(A)$, the presheaf of equivalence classes of families of stable graded A-modules.

In the coprime case, $\left(L^{s} / / \widetilde{G}, \mathscr{R}\right)$ represents $\mathfrak{R} \widetilde{\mathfrak{M}}_{\alpha}^{s}(A)$.

Example 2.4. Maybe the most canonical of all characters is the one defined by $\theta_{p}=-\operatorname{dim} V_{q}, \theta_{q}=\operatorname{dim} V_{p}$ and all other $\theta_{i}=0$. We call it the extremal character. For this character, (semi)stability reads

$$
\operatorname{dim} W_{p} \operatorname{dim} V_{q}(\leq) \operatorname{dim} W_{q} \operatorname{dim} V_{p},
$$

or, equivalently,

or

$$
\frac{\operatorname{dim} W_{p}}{\operatorname{dim} W_{q}}(\leq) \frac{\operatorname{dim} V_{p}}{\operatorname{dim} V_{q}}
$$

$$
\frac{\operatorname{dim} W_{p}}{\operatorname{dim} V_{p}}(\leq) \frac{\operatorname{dim} W_{q}}{\operatorname{dim} V_{q}}
$$

For example, stability implies that $V_{p}$ generates $V$ as an $A$-module. 
Definition 2.5. We call the $[p, q]$-graded $A$-module $M$ (semi)stable if the corresponding point $\mu$ in $L^{1}=\operatorname{Hom}_{\mathrm{gr}}(\mathfrak{m}$, $\operatorname{End}(M))$ is (semi)stable with respect to the linearization of $\widetilde{G}$ given by the extremal character.

Example 2.6. Another canonical character is the determinant of the action of $G$ on $L^{1}$. It has

$$
\theta_{i}=\sum_{j<i} \operatorname{dim} A_{i-j} \operatorname{dim} V_{j}-\sum_{j>i} \operatorname{dim} A_{j-i} \operatorname{dim} V_{j},
$$

and gives rise to the (semi)stability condition

$$
\sum_{i<j} \operatorname{dim} A_{j-i} \operatorname{dim} W_{i} \operatorname{dim} V_{j}(\leq) \sum_{i<j} \operatorname{dim} A_{j-i} \operatorname{dim} W_{j} \operatorname{dim} V_{i} .
$$

\section{Moduli of sheaves}

We will now assume that $A=\bigoplus_{n \geq 0} \Gamma(Y, \mathcal{O}(n))$ for a connected projective scheme $Y$.

3A. The adjoint of the truncation functor. For a scheme $T$, we denote the projection $Y \times T \rightarrow T$ by $\pi_{T}$.

Let $T$ be a scheme and $\mathscr{F}_{T}$ a coherent sheaf on $Y \times T$. Then

$$
\Gamma_{[p, q]}{ }^{\mathscr{F}} T=\bigoplus_{i=p}^{q} \pi_{T *}(\mathscr{F}(i))
$$

is a graded sheaf of coherent $O_{T}$-modules with $A$-module structure.

\section{Proposition 3.1. The functor}

$\Gamma_{[p, q]}:$ (coherent sheaves of $\mathrm{O}_{Y \times T}$-modules)

$\longrightarrow\left([p, q]\right.$-graded coherent sheaves of $A \otimes \mathcal{O}_{T}$-modules $)$

has a left adjoint, which we shall denote by $\mathscr{Y}$. The functor $\mathscr{S}$ commutes with arbitrary base change.

Proof. First note that graded coherent $A \otimes \mathcal{O}_{T}$-modules concentrated in the interval $[p, q]$ form an abelian category with kernels, cokernels, images and direct sums constructed degreewise, and that $\Gamma_{[p, q]}$ is an additive functor so that the statement makes sense.

Then, by the claimed compatibility with base change, we may assume that $T$ is affine, $T=\operatorname{Spec} B$.

Let $M$ be a graded $A \otimes B$-module concentrated in the interval [ $p, q]$, and let

$$
\bigoplus_{j} A\left(-m_{j}\right) \otimes B \longrightarrow \bigoplus_{i} A\left(-n_{i}\right) \otimes B \longrightarrow M \longrightarrow 0
$$


be a presentation of $M$ (by graded homomorphisms) as a graded $A \otimes B$-module. Assume that all $n_{i}$ are in the interval $[p, q]$.

Define $\mathscr{Y} M$ to be the cokernel in the diagram of $0_{Y \times T}$-modules

$$
\bigoplus_{m_{j} \in[p, q]} \mathcal{O}_{Y \times T}\left(-m_{j}\right) \longrightarrow \bigoplus_{i} \mathcal{O}_{Y \times T}\left(-n_{i}\right) \longrightarrow \mathscr{S} \longrightarrow 0
$$

where the first sum extends only over those indices $j$ such that $m_{j}$ is in the interval $[p, q]$. Let us prove that $\mathscr{Y} M$ defined in this way satisfies

$$
\operatorname{Hom}_{\mathscr{O}_{Y \times T}}(\mathscr{Y} M, \mathscr{F})=\operatorname{Hom}_{A \otimes B}^{\mathrm{gr}}\left(M, \Gamma_{[p, q]} \mathscr{F}\right),
$$

for all $O_{Y \times T}$-modules $\mathscr{F}$. Given such $\mathscr{F}$, consider the commutative diagram

$$
\begin{gathered}
\operatorname{Hom}_{\mathscr{O}_{Y \times T}}\left(\bigoplus_{i} \mathcal{O}_{Y \times T}\left(-n_{i}\right), \mathscr{F}\right) \longrightarrow \operatorname{Hom}_{\mathscr{O}_{Y \times T}}\left(\bigoplus_{m_{j} \in[p, q]} \mathcal{O}_{Y \times T}\left(-m_{j}\right), \mathscr{F}\right) \\
\| \\
\operatorname{Hom}_{A \otimes B}^{\mathrm{gr}}\left(\bigoplus_{i} A\left(-n_{i}\right) \otimes B, \Gamma_{[p, q]} \mathscr{F}\right) \longrightarrow \operatorname{Hom}_{A \otimes B}^{\mathrm{gr}}\left(\bigoplus_{j} A\left(-m_{j}\right) \otimes B, \Gamma_{[p, q]} \mathscr{F}\right)
\end{gathered}
$$

This diagram induces an equality of the kernels of the horizontal maps, and these kernels are the two sides of (9), thus proving (9).

To prove that the adjoint functor $\mathscr{Y}$ commutes with base change, consider a base change diagram

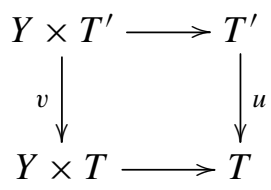

and note that $\Gamma_{[p, q]} \circ v_{*}=u_{*} \circ \Gamma_{[p, q]}^{\prime}$ in obvious notation. It follows that for the adjoint functors we have the equality $v^{*} \circ \mathscr{S}=\mathscr{S}^{\prime} \circ u^{*}$, which is the claimed compatibility with base change.

3B. Open immersion. Fix a numeric polynomial $\alpha(t)=a_{0}\left(\begin{array}{l}t \\ 0\end{array}\right)+\cdots+a_{k}\left(\begin{array}{l}t \\ k\end{array}\right)$.

Let $\mathfrak{U}$ be a finite type open substack of the algebraic stack of coherent sheaves on $Y$ with Hilbert polynomial $\alpha(t)$.

For $q>p>0$ let $\mathfrak{M o d}_{\alpha}^{[p, q]}(A)$ be the algebraic stack of $[p, q]$-graded $A$-modules of dimension $\left.\alpha\right|_{[p, q]}$. Recall that $\operatorname{Mod}_{\alpha}^{[p, q]}(A)=[M C(L) / G]$, in the notation of Section 1.

Proposition 3.2. Given $\mathfrak{U}$, there exists $p$ such that for all $q>p$ the functor $\Gamma_{[p, q]}$ defines a morphism of algebraic stacks

$$
\Gamma_{[p, q]}: \mathfrak{U} \longrightarrow \operatorname{Mod}_{\alpha}^{[p, q]}(A) .
$$


If $q$ is sufficiently large, then $\Gamma_{[p, q]}$ is a monomorphism of stacks.

Proof. Let $p$ be large enough such that every sheaf in $\mathfrak{U}$ is Castelnuovo-Mumford $p$-regular. Then, for every $i \geq p$, the sheaf $\pi_{T *} \mathscr{F}(i)$ is locally free of rank $\alpha(i)$ on $T$. Hence $\Gamma_{[p, q]} \mathscr{F}$ is a $\mathfrak{M o d}_{\alpha}^{[p, q]}(A)$-family over $T$, and we have the required morphism of stacks.

Now let, in addition, $q$ be large enough for $\mathrm{O}_{Y}(q-p)$ to be Castelnuovo-Mumford regular. Then $\Gamma_{[p, q]}$ is a monomorphism of stacks because for every family of $p$ regular sheaves $\mathscr{F}_{\mathcal{F}}$, the adjunction map $\mathscr{Y}\left(\Gamma_{[p, q]} \mathscr{F}\right) \rightarrow \mathscr{F}$ is an isomorphism. See [Álvarez-Cónsul and King 2007], Theorem 3.4 and Proposition 4.1, for a proof of a similar statement. In our context, we may proceed as follows:

First note that we may assume that the parameter scheme $T$ is affine, $T=\operatorname{Spec} B$, as in the proof of Proposition 3.1.

Let $V=\Gamma(Y, \mathscr{F}(p))$, and $\mathscr{G}$ the kernel in:

$$
0 \longrightarrow \mathscr{G} \longrightarrow V \otimes_{B} \mathrm{O}_{Y}(-p) \longrightarrow \mathscr{F} \longrightarrow 0 .
$$

Then the fact that $\mathscr{O}_{Y}(q-p)$ is regular implies that $\mathscr{G}$ is $q$-regular. (See Lemma 3.3 in [Álvarez-Cónsul and King 2007].) Let $W=\Gamma(Y, \mathscr{G}(q))$, so that we have a surjection $W \otimes_{B} \mathscr{O}_{Y}(-q) \rightarrow \mathscr{G}$ and a presentation of $\mathscr{F}$ :

$$
W \otimes_{B} \mathrm{O}_{Y}(-q) \longrightarrow V \otimes_{B} \mathrm{O}_{Y}(-p) \longrightarrow \mathscr{F}^{\longrightarrow} \longrightarrow 0
$$

We remark that $q$-regularity of $\varphi$ implies that this sequence stays exact after twisting by $O_{Y}(i)$ and taking global sections for all $i \geq q$. Thus the sequence of graded $A \otimes B$-modules

$$
W \otimes A(-q) \longrightarrow V \otimes A(-p) \longrightarrow \Gamma_{\geq p} \mathscr{F} \longrightarrow 0
$$

is exact in degrees $\geq q$. We can construct from this a presentation of $\Gamma_{\geq p} \mathscr{F}$ by adding some relations whose degrees are between $p$ and $q$. Then we can turn this presentation of $\Gamma_{\geq p} \mathscr{F}$ into a presentation of $\Gamma_{[p, q]} \mathscr{F}_{\mathscr{F}}$ by adding relations in degrees larger than $q$. These extra relations in degrees larger than $q$ are ignored when constructing $\mathscr{S}\left(\Gamma_{[p, q]}{ }_{F}\right)$; see the proof of Proposition 3.1. The extra relations of degree between $p$ and $q$ do not affect the cokernel in Equation (8). We conclude that we have a presentation

$$
W \otimes_{B} \mathrm{O}_{Y}(-q) \longrightarrow V \otimes_{B} \mathrm{O}_{Y}(-p) \longrightarrow \mathscr{S}\left(\Gamma_{[p, q]}{ }^{\mathscr{F}}\right) \longrightarrow 0 .
$$

This proves that $\mathscr{S}\left(\Gamma_{[p, q]} \mathscr{F}\right)=\mathscr{F}$.

Proposition 3.3. For $q \gg p \gg 0$ the morphism $\Gamma_{[p, q]}: \mathfrak{U} \rightarrow \mathfrak{M o d}_{\alpha}^{[p, q]}(A)$ is étale. 
Proof. Let $A^{\prime} \rightarrow A \rightarrow \mathbb{C}$ be a small extension of pointed $\mathbb{C}$-algebras (not differential graded). Let $T=\operatorname{Spec} A$ and $T^{\prime}=\operatorname{Spec} A^{\prime}$. Consider a 2-commutative diagram

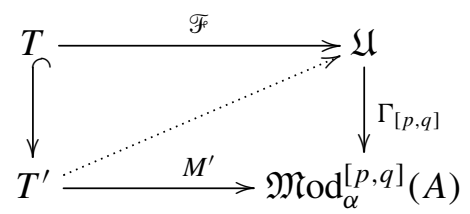

of solid arrows. We have to prove that the dotted arrow exists, uniquely, up to a unique 2-isomorphism. This follows from standard deformation-obstruction theory. We need that $\Gamma_{[p, q]}$ induces a bijection on deformation spaces and an injection on obstruction spaces (associated to the above diagram). It is well known that deformations of $\mathscr{F}$ are classified by $\operatorname{Ext}_{\mathscr{O}_{Y}}^{1}(\mathscr{F}, \mathscr{F})$, and obstructions are contained in $\operatorname{Ext}_{\mathscr{O}_{Y}}^{2}(\mathscr{F}, \mathscr{F})$. We saw in Corollary 1.18 that deformations of $\left.M^{\prime}\right|_{T}$ are classified by $\operatorname{Ext}_{A}^{1}(M, M)_{\mathrm{gr}}$ and obstructions are contained in $\operatorname{Ext}_{A}^{2}(M, M)_{\mathrm{gr}}$, where $M=\Gamma_{[p, q]}(\mathscr{F})$. It is proved in [Ciocan-Fontanine and Kapranov 2001], (4.3.3.a) and (4.3.4) that for fixed $i$, there exist $q \gg p \gg 0$ such that $\operatorname{Ext}_{\mathscr{O}_{Y}}^{i}\left(\mathscr{F}_{\mathscr{F}}, \mathscr{F}\right)=$ $\operatorname{Ext}_{A}^{i}\left(\Gamma_{[p, q]} \mathscr{F}^{\mathscr{F}}, \Gamma_{[p, q]} \mathscr{F}_{\mathrm{Fr}}\right.$. (Note that the assumption in [Ciocan-Fontanine and Kapranov 2001] that $Y$ be smooth is not used for this result. It is only used to exchange quantifiers: namely to get uniform $p$ and $q$, which work for all $i \geq 0$.)

Corollary 3.4. For $q \gg p \gg 0$ the morphism $\Gamma_{[p, q]}: \mathfrak{U} \rightarrow \mathfrak{M o d}_{\alpha}^{[p, q]}(A)$ is an open immersion.

3C. Stable sheaves. Let $Y$ be a connected projective scheme. We denote the Hilbert polynomial of a coherent sheaf $\mathscr{F}$ on $Y$ by $h(\mathscr{F}, t)=h(\mathscr{F})$.

For our purposes, the following characterization of stability of $\mathscr{F}$ is most useful.

Definition 3.5. The sheaf $\mathscr{F}$ is (semi)stable if and only if for every proper subsheaf $0<\mathscr{F}^{\prime}<\mathscr{F}$ we have

$$
\frac{h\left(\mathscr{F}^{\prime}, p\right)}{h\left(\mathscr{F}^{\prime}, p\right)}(\leq) \frac{h\left(\mathscr{F}^{\prime}, q\right)}{h\left(\mathscr{F}^{\prime}, q\right)} \quad \text { for } q \gg p \gg 0 .
$$

(As usual, this means the strict inequality for "stable" and the weak inequality for "semistable".)

The condition needs only to be checked for saturated subsheaves. (A subsheaf is saturated if the corresponding quotient is pure of the same dimension as $\mathscr{F}$.)

Remark 3.6. We can say, informally, that the limiting slope of the quotient of Hilbert polynomials $h\left(\mathscr{F}^{\prime}\right) / h(\mathscr{F})$ is $(\geq)$, for all proper saturated subsheaves.

This stability condition looks very similar to the condition given by the extremal character for $A$-modules (see Example 2.4), but to relate the two notions is not completely trivial. 
Theorem 3.7. Given $\mathfrak{U}$, it is possible to choose $q \gg p \gg 0$ in such a way that the following holds: if $\mathscr{F}$ is a $\mathfrak{U}$-sheaf, then $\mathscr{F}$ is a (semi)stable sheaf if and only if $M=\Gamma_{[p, q]} \mathscr{F}_{F}$ is a (semi)stable graded A-module (Definition 2.5).

Proof. By Grothendieck's lemma (see [Huybrechts and Lehn 1997], Lemma 1.7.9), the family $\mathfrak{U}^{\prime}$ of all saturated destabilizing subsheaves of all sheaves in $\mathfrak{U}$ is bounded. We choose $p$ large enough to ensure that all sheaves in $\mathfrak{U}$ and $\mathfrak{U}^{\prime}$ are $p$-regular. Note that the sheaves in $\mathfrak{U}^{\prime}$ have only finitely many Hilbert polynomials. So we can choose $q \gg p \gg 0$ in such a way that the limiting slope of all quotients of all Hilbert polynomials involved is measured correctly by $p$ and $q$.

Additionally, we choose $p$ and $q$ sufficiently large as explicated in [ÁlvarezCónsul and King 2007]. (This choice is only needed for the "converse", below.)

Let us first suppose that $M$ is (semi)stable and prove that $\mathscr{F}$ is (semi)stable. So let $0 \subsetneq \mathscr{F}^{\prime} \subsetneq \mathscr{F}$ be a saturated subsheaf. We wish to prove, of course, that $\mathscr{F}^{\prime}$ does not violate (semi)stability of $\mathscr{F}$. So let us assume it does. Then by our choices, both $\mathscr{F}^{\prime}$ and $\mathscr{F}$ are $p$-regular.

Since $\Gamma_{[p, q]}$ is left exact, we get a graded submodule

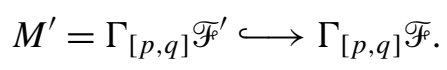

Moreover, $0 \subsetneq M^{\prime} \subsetneq M$, as $\mathscr{F}^{\prime}=\mathscr{Y} M^{\prime}$ because $\mathscr{F}^{\prime}$ is $p$-regular. Since $M=\Gamma_{[p, q]} \mathscr{F}$ is (semi)stable, we know that

$$
\frac{\operatorname{dim} \Gamma\left(Y, \mathscr{F}^{\prime}(p)\right)}{\operatorname{dim} \Gamma(Y, \mathscr{F}(p))}(\leq) \frac{\operatorname{dim} \Gamma\left(Y, \mathscr{F}^{\prime}(q)\right)}{\operatorname{dim} \Gamma(Y, \mathscr{F}(q))} .
$$

By $p$-regularity, this implies that

$$
\frac{h\left(\mathscr{F}^{\prime}, p\right)}{h\left(\mathscr{F}^{\prime}, p\right)}(\leq) \frac{h\left(\mathscr{F}^{\prime}, q\right)}{h\left(\mathscr{F}^{\prime}, q\right)},
$$

and so $\mathscr{F}^{\prime}$ does not violate (semi)stability, a contradiction.

Conversely, assume that $\mathscr{F}$ is (semi)stable. If $0<M^{\prime}<M$ is a (semi)stability violating submodule, then $\left(M_{p}^{\prime}, M_{q}^{\prime}\right) \subset\left(M_{p}, M_{q}\right)$ is a Kronecker submodule in the sense of [Álvarez-Cónsul and King 2007]. To prove that $\left(M_{p}^{\prime}, M_{q}^{\prime}\right) \neq(0,0)$, note that $\Gamma_{[p, q]} \mathscr{F}$ does not have any nontrivial submodules which vanish in the top degree $q$. (This is an elementary fact about sheaves on projective schemes.) To prove that $\left(M_{p}^{\prime}, M_{q}^{\prime}\right) \neq\left(M_{p}, M_{q}\right)$, note that $\Gamma_{[p, q]} \mathscr{F}$ is generated in the lowest degree $p$, by $p$-regularity of $\mathscr{F}$.

Thus, applying [ibid., Theorem 5.10], we see that $M^{\prime}$ does not violate (semi)stability, a contradiction.

3D. Moduli of sheaves. Let $\alpha(t)$ be a Hilbert polynomial. Let $\mathfrak{U}^{s s}$ be the bounded family of all semistable sheaves with Hilbert polynomial $\alpha(t)$. Choose $q \gg p \gg 0$ as prescribed by Theorem 3.7 for $\mathfrak{U}^{s s}$. 
Let $U^{s} \subset U^{s s}$ be the moduli spaces of stable (respectively, semistable) sheaves on $Y$ with Hilbert polynomial $\alpha(t)$.

Corollary 3.8. We have a commutative diagram of open immersions of schemes.

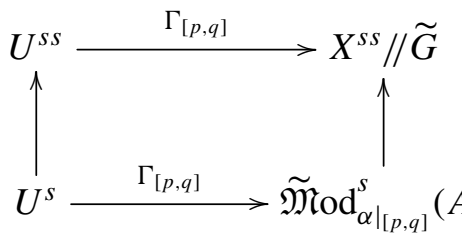

The two schemes in the top row are projective. Hence, $U^{s s}$ is a union of connected components of $X^{s s} / / \widetilde{G}$.

In the case where $\alpha$ is primitive, we have $U^{s}=U^{s s}$, and so $U^{s}$ is a union of components of $\tilde{\mathfrak{M}}_{\left.\mathrm{od}_{\alpha}\right|_{[p, q]}}$ via the functor $\Gamma_{[p, q]}$.

Remark 3.9. Assume we are in the primitive case. Then $U^{s} \subset L^{s} / / \widetilde{G}$ is a closed subscheme of the smooth scheme $L^{s} / / \widetilde{G}$, cut out by the descended Maurer-Cartan equation $d x+\frac{1}{2}[x, x]=0$. This gives rather explicit equations for $U^{s}$ inside a smooth scheme. Note that we do not prove that $L^{s} / / \widetilde{G}$ is projective, in the primitive case.

3E. An amplification. By using three integers $q \gg p^{\prime} \gg p \gg 0$, we can describe the image of $\Gamma_{[p, q]}: \mathfrak{U}^{s} \rightarrow \mathfrak{M o d}_{\left.\alpha\right|_{[p, q]}}(A)$ explicitly.

We denote by $\mathfrak{M o d}_{\left.\alpha\right|_{[p, q]}}(A)^{\prime} \subset \operatorname{Mod}_{\left.\alpha\right|_{[p, q]}}(A)$ the open substack of graded $A$ modules which are generated in degree $p$.

Theorem 3.10. Let $\mathfrak{U}$ be, as above, a bounded open family of sheaves on $Y$. Then for $q \gg p^{\prime} \gg p \gg 0$, the functor $\Gamma_{[p, q]}$ induces an open immersion

$$
\Gamma_{[p, q]}: \mathfrak{U} \longrightarrow \operatorname{Mod}_{\left.\alpha\right|_{[p, q]}}(A)^{\prime},
$$

and the image of $\mathfrak{U}^{s}\left(\mathfrak{U}^{s s}\right)$ is equal to the locus of modules whose truncation into the interval $\left[p^{\prime}, q\right]$ is (semi)stable.

Proof. The first claim is clear: $p$-regularity of $\mathscr{F}$ implies that $\Gamma_{[p, q]} \mathscr{F}_{\mathscr{F}}$ is generated in degree $p$.

The fact that $\mathfrak{U}^{s}\left(\mathfrak{U}^{s s}\right)$ is contained in the $\left[p^{\prime}, q\right]$-(semi)stable locus follows from Theorem 3.7.

Let $M$ be an $A$-module concentrated in degrees $[p, q]$, generated in degree $p$, and of dimension $\left.\alpha\right|_{[p, q]}$. Then we will use Gotzmann persistence to prove that $\mathscr{F}=\mathscr{Y}(M)$ has Hilbert polynomial $\alpha$, and we will make sure that all $\mathscr{Y}(M)$ obtained in this way are $p^{\prime}$-regular. This will imply that $M_{\left[p^{\prime}, q\right]}=\Gamma_{\left[p^{\prime}, q\right]} \mathscr{F}$, and we can again apply Theorem 3.7 to deduce that if $M_{\left[p^{\prime}, q\right]}$ is (semi)stable, then $\mathscr{F}$ is (semi)stable. 
We briefly recall the persistence theorem (see [Gotzmann 1978] and [Gasharov 1997], especially Theorem 4.2). First, for integers $a \geq 0$ and $t \geq 1$, there exist unique integers $m_{t}>m_{t-1}>\cdots>m_{1} \geq 0$, such that $a=\sum_{i=1}^{t}\left(\begin{array}{c}m_{i} \\ i\end{array}\right)$. Then one defines $a^{\langle t\rangle}=\sum_{i=1}^{t}\left(\begin{array}{c}m_{i}+1 \\ i+1\end{array}\right)$. One significance of this definition is the following: if $\mathscr{E}$ is a coherent sheaf of $\mathrm{O}_{Y}$-modules, such that $\mathscr{E}(p)$ is globally generated, and if $h(t)$ is the Hilbert polynomial of $\mathscr{E}$, then $h(t+1)=h(t)^{\langle t-p\rangle}$, for $t \gg 0$. The persistence theorem says the following:

Suppose $A$ is a graded $\mathbb{C}$-algebra, generated in degree 1 , with relations in degree $\leq r$ for an integer $r \geq 1$. Let $M$ be a graded $A$-module and $G$ a finite-dimensional graded $\mathbb{C}$-vector space such that the following sequence of graded $A$-modules is exact:

$$
0 \longrightarrow K \longrightarrow A \otimes_{\mathbb{C}} G \longrightarrow M \longrightarrow 0 .
$$

(i) (Macaulay bound) If $\operatorname{deg} G \leq p$, then $\operatorname{dim} M_{d+1} \leq\left(\operatorname{dim} M_{d}\right)^{\langle d-p\rangle}$ for all $d \geq p+1$. Moreover, there exists a $d$ such that $\operatorname{dim} M_{d^{\prime}+1}=\left(\operatorname{dim} M_{d^{\prime}}\right)^{\left\langle d^{\prime}-p\right\rangle}$, for all $d^{\prime} \geq d$.

(ii) (persistence) If in addition $K$ is generated in degree less than or equal to $r^{\prime}$, where $r^{\prime} \geq p+r$, and if $\operatorname{dim} M_{d+1}=\left(\operatorname{dim} M_{d}\right)^{\langle d-p\rangle}$ for some $d \geq r^{\prime}$, then $\operatorname{dim} M_{d^{\prime}+1}=\left(\operatorname{dim} M_{d^{\prime}}\right)^{\left\langle d^{\prime}-p\right\rangle}$ for all $d^{\prime} \geq d$.

We may assume that $\alpha(t+1)=\alpha(t)^{\langle t-p\rangle}$, for all $t \geq p$.

Now let $M$ be an $A$-module in $[p, q]$ of dimension $\left.\alpha\right|_{[p, q]}$ and generated in degree $p$. We have the exact sequence

$$
0 \longrightarrow K \longrightarrow A_{[0, q-p]} \otimes M_{p} \longrightarrow M \longrightarrow 0,
$$

where the kernel $K$ exists (at most) in degrees $[p+1, q]$. Let $\widetilde{K} \subset A \otimes M_{p}$ be the submodule generated by $K$, and let $\widetilde{M}$ be the quotient

$$
0 \longrightarrow \widetilde{K} \longrightarrow A \otimes M_{p} \longrightarrow \tilde{M} \longrightarrow 0 .
$$

Thus $\widetilde{K}$ is generated in degree $\leq q$.

Our first claim is that $\widetilde{K}$ is actually generated in degree $p+1$. We will do this by descending induction. So suppose $\widetilde{K}$ is generated in degree $\leq r^{\prime}$ for $p+1<r^{\prime} \leq q$, but not in degree $\leq r^{\prime}-1$. Then let $\widetilde{K}^{\prime}<\widetilde{K}$ be the submodule generated by the degree $\leq r^{\prime}-1$ part of $\widetilde{K}$. Let $\widetilde{M}^{\prime}=\left(A \otimes M_{p}\right) / \widetilde{K}^{\prime}$ be the quotient. Then we have

$$
\left(\operatorname{dim} \tilde{M}_{r^{\prime}-1}^{\prime}\right)^{\left\langle r^{\prime}-1-p\right\rangle} \geq \tilde{M}_{r^{\prime}}^{\prime}>\tilde{M}_{r^{\prime}}=\left(\tilde{M}_{r^{\prime}-1}\right)^{\left\langle r^{\prime}-1-p\right\rangle},
$$

which implies $\operatorname{dim} \tilde{M}_{r^{\prime}-1}^{\prime}>\operatorname{dim} \widetilde{M}_{r^{\prime}-1}$, which is absurd, as these two spaces are equal. Thus $\widetilde{K}$ is, indeed, generated in degrees $\leq r^{\prime}-1$, and we conclude that it is, in fact, generated in degree $p+1$.

Now, the persistence theorem implies that $\operatorname{dim} \widetilde{M}_{t+1}=\operatorname{dim} \widetilde{M}_{t}^{\langle t-p\rangle}$ for $t>p+r$. 
As $\mathscr{S}(M)$ is the sheaf associated to $\tilde{M}$, this implies that the Hilbert polynomial of $\mathscr{Y}(M)$ is equal to $\alpha$, as claimed.

We remark that the family of all $A$-modules generated in degree $p$ by $\alpha(p)$ elements, whose relations are in degree $p+1$, is bounded. Therefore, we can choose $p^{\prime}>p$ in such a way that all sheaves associated to such modules are $p^{\prime}$ regular. This will imply that all $\mathscr{S}(M)$ obtained from $\mathfrak{M o d}_{\left.\alpha\right|_{[p, q]}}(A)^{\prime}$ are $p^{\prime}$-regular.

It remains to prove that a suitable choice of $p^{\prime}$ will assure that the truncation of $M$ into the interval $\left[p^{\prime}, q\right]$ is equal to $\Gamma_{\left[p^{\prime}, q\right]} \mathscr{F}$, where $\mathscr{F}=\mathscr{Y}(M)$.

Now, the canonical map $\widetilde{M} \rightarrow \Gamma_{\geq p} \mathscr{F}$ is an isomorphism in sufficiently high degree. But as the family of all $\widetilde{M}$ which occur is bounded, there exists a uniform $p^{\prime}$ which will assure that $\left.\widetilde{M}\right|_{\geq p^{\prime}} \rightarrow \Gamma_{\geq p^{\prime}} \mathscr{F}$ is an isomorphism. This finishes the proof of the last remaining fact that $\left.M\right|_{\left[p^{\prime}, q\right]}=\Gamma_{\left[p^{\prime}, q\right]} \mathscr{S}(M)$.

Corollary 3.11. We have

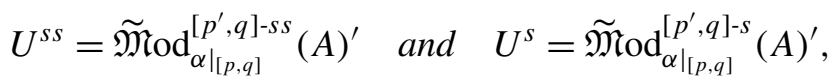

in obvious notation. In the primitive case, all four schemes are equal.

Remark 3.12. If an $A$-module in $[p, q]$ is stable (not just semistable), then it is generated in degree $p$. Thus $U^{s}$ can also be described as the scheme of modules in the interval $[p, q]$ of dimension $\left.\alpha\right|_{[p, q]}$ which are stable and whose truncation into the interval $\left[p^{\prime}, q\right]$ is also stable.

\section{Derived moduli of sheaves}

Finally, we will construct the differential graded moduli scheme of stable sheaves on the projective variety $Y$. From now on, we have to assume that $Y$ is smooth. Let $\alpha(t)$ be a numerical polynomial, and $p \gg 0$. For simplicity, let us assume that $\alpha(t)$ is primitive.

Definition 4.1. A family of coherent sheaves on $Y$ of Hilbert polynomial $\alpha(t)$, parametrized by the differential graded scheme $\left(T, \mathscr{R}_{T}\right)$, is a pair $(E, \mu)$, where $E$ is a graded quasicoherent sheaf

$$
E=\bigoplus_{i \geq p} E_{i}
$$

on $T$ and each $E_{i}$ is a vector bundle of rank $\operatorname{rk} E_{i}=\alpha(i)$. Moreover, $\mu$ is a "unital" Maurer-Cartan element in the differential graded Lie algebra

$$
\Gamma\left(T, \mathscr{H o m}_{\mathrm{gr}}\left(A^{\otimes \geq 1}, \mathscr{E} n d_{\mathscr{O}_{T}} E\right) \otimes_{\mathbb{O}_{T}} \mathscr{R}_{T}\right) ;
$$

in other words a graded unital $\mathscr{R}_{T}$-linear $A_{\infty}$-action of $A \otimes \mathscr{R}_{T}$ on $E \otimes_{\mathcal{O}_{T}} \mathscr{R}_{T}$.

We denote the functor of equivalence classes (see Definition 1.9) of simple such families by $\mathfrak{R} \tilde{\mathfrak{M}}_{\alpha}^{\mathrm{sp}}\left(\mathrm{O}_{Y}\right)$. 
If for every point $P: \operatorname{Spec} \mathbb{C} \rightarrow T$, the associated coherent sheaf on $Y$ is (semi)stable, then the family $(E, \mu)$ is a (semi)stable family.

Lemma 4.2. We have

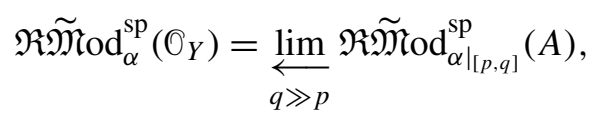

as set-valued presheaves on the category of differential graded schemes.

Proof. Obvious.

Corollary 4.3. The functor $\mathfrak{\Re} \tilde{\mathfrak{M}}_{\alpha}^{\mathrm{sp}}\left(\mathrm{O}_{Y}\right)$ is represented by the projective limit of differential graded algebraic spaces

$$
\mathfrak{R} \tilde{\mathfrak{M}}_{\alpha}^{\mathrm{sp}}(A)=\lim _{q \gg p} \mathfrak{R} \tilde{\mathfrak{M}}_{\mathrm{od}_{\alpha}}^{\mathrm{sp}}(A),
$$

Proposition 4.4. The projective limit

$$
\lim _{q \gg p} \mathfrak{R} \tilde{\mathfrak{M}}_{\left.\alpha\right|_{[p, q]}}^{\mathrm{sp}}(A)
$$

stabilizes as far as quasiisomorphism is concerned. More precisely, for $q^{\prime} \gg q \gg p$ the morphism

$$
\mathfrak{R} \tilde{\mathfrak{M}}_{\left.\alpha\right|_{\left[p, q^{\prime}\right]} ^{\mathrm{sp}}}^{\mathrm{sp}}(A) \longrightarrow \mathfrak{R} \tilde{\mathfrak{M}}_{\left.\alpha\right|_{[p, q]}}^{\mathrm{sp}}(A)
$$

is a quasiisomorphism of differential graded algebraic spaces.

Proof. Here we use that $Y$ is smooth to deduce that

$$
\operatorname{Ext}_{\bigcirc_{Y}}^{i}(E, E)=\operatorname{Ext}_{A}^{i}\left(\Gamma_{[p, q]} E, \Gamma_{[p, q]} E\right),
$$

for $q \gg p$. Then we use the fact that if $\pi_{0}$ agrees and tangent complex cohomologies agree, then a morphism of differential graded schemes is a quasiisomorphism.

Corollary 4.5. If $q \gg p$, then $\mathfrak{R} \tilde{\mathfrak{M}} \mathrm{d}_{\alpha}^{\mathrm{sp}}\left(\mathcal{O}_{Y}\right)$ is quasiisomorphic to $\mathfrak{R} \tilde{\mathfrak{M}}_{\left.\alpha\right|_{[p, q]}}^{\mathrm{sp}}(A)$. Moreover, $\mathfrak{R} \tilde{M}^{s} d_{\alpha}^{s}\left(O_{Y}\right)$ is an open and closed differential graded subscheme of $\mathfrak{R} \tilde{\mathfrak{M}}_{\left.\alpha\right|_{[p, q]}}^{\mathrm{sp}}(A)$.

\section{References}

[Álvarez-Cónsul and King 2007] L. Álvarez-Cónsul and A. King, "A functorial construction of moduli of sheaves”, Invent. Math. 168:3 (2007), 613-666. MR 2008d:14015 Zbl 1137.14026

[Behrend and Fantechi 1997] K. Behrend and B. Fantechi, "The intrinsic normal cone", Invent. Math. 128:1 (1997), 45-88. MR 98e:14022 Zbl 0909.14006

[Ciocan-Fontanine and Kapranov 2001] I. Ciocan-Fontanine and M. Kapranov, "Derived Quot schemes”, Ann. Sci. École Norm. Sup. (4) 34:3 (2001), 403-440. MR 2002k:14003 Zbl 1050.14042

[Ciocan-Fontanine and Kapranov 2009] I. Ciocan-Fontanine and M. Kapranov, "Virtual fundamental classes via dg-manifolds", Geom. Topol. 13:3 (2009), 1779-1804. MR 2010e:14012 Zbl 1159.14002 
[Gasharov 1997] V. Gasharov, "Extremal properties of Hilbert functions", Illinois J. Math. 41:4 (1997), 612-629. MR 98m:13024 Zbl 0908.13009

[Gotzmann 1978] G. Gotzmann, "Eine Bedingung für die Flachheit und das Hilbertpolynom eines graduierten Ringes”, Math. Z. 158:1 (1978), 61-70. MR 58 \#641 Zbl 0352.13009

[Huybrechts and Lehn 1997] D. Huybrechts and M. Lehn, The geometry of moduli spaces of sheaves, Aspects of Mathematics E31, Vieweg, Braunschweig, 1997. MR 98g:14012 Zbl 0872.14002

[King 1994] A. D. King, "Moduli of representations of finite-dimensional algebras", Quart. J. Math. Oxford Ser. (2) 45:180 (1994), 515-530. MR 96a:16009 Zbl 0837.16005

[Loday 1992] J.-L. Loday, Cyclic homology, Grundlehren der Mathematischen Wissenschaften 301, Springer, Berlin, 1992. MR 94a:19004 Zbl 0780.18009

[Lurie 2009] J. Lurie, Higher topos theory, Annals of Mathematics Studies 170, Princeton University Press, Princeton, NJ, 2009. MR 2010j:18001 Zbl 1175.18001

[Manetti 1999] M. Manetti, "Deformation theory via differential graded Lie algebras", pp. 21-48 in Seminari di geometria algebrica (Pisa, 1998-1999), Scuola Normale Superiore, Pisa, 1999. MR 1754793

[Simpson 1994] C. T. Simpson, "Moduli of representations of the fundamental group of a smooth projective variety, I", Inst. Hautes Études Sci. Publ. Math. 79:79 (1994), 47-129. MR 96e:14012 Zbl 0891.14005

[Thomas 2000] R. P. Thomas, "A holomorphic Casson invariant for Calabi-Yau 3-folds, and bundles on K3 fibrations", J. Differential Geom. 54:2 (2000), 367-438. MR 2002b:14049 Zbl 1034.14015

[Toën and Vaquié 2007] B. Toën and M. Vaquié, "Moduli of objects in dg-categories", Ann. Sci. École Norm. Sup. (4) 40:3 (2007), 387-444. MR 2009m:18015 Zbl 1140.18005

[Toën and Vezzosi 2004] B. Toën and G. Vezzosi, "From HAG to DAG: derived moduli stacks", pp. 173-216 in Axiomatic, enriched and motivic homotopy theory, NATO Sci. Ser. II Math. Phys. Chem. 131, Kluwer Acad. Publ., Dordrecht, 2004. MR 2005f:14001 Zbl 1076.14002

[Toën and Vezzosi 2005] B. Toën and G. Vezzosi, "Homotopical algebraic geometry, I: Topos theory", Adv. Math. 193:2 (2005), 257-372. MR 2007b:14038 Zbl 1120.14012

[Toën and Vezzosi 2008] B. Toën and G. Vezzosi, Homotopical algebraic geometry, II: Geometric stacks and applications, vol. 193, Mem. Amer. Math. Soc. 902, Amer. Math. Soc., Providence, RI, 2008. MR 2009h:14004

[Weibel 1994] C. A. Weibel, An introduction to homological algebra, Cambridge Studies in Advanced Mathematics 38, Cambridge University Press, Cambridge, 1994. MR 95f:18001 Zbl 0797.18001

Communicated by Mikhail Kapranov

Received 2010-04-28 Revised 2012-09-03 Accepted 2012-11-21

behrend@math.ubc.ca

ciocan@math.umn.edu

jhhwang@math.ubc.ca

michael.a.j.rose@gmail.com
Department of Mathematics, University of British Columbia, 1984 Mathematics Road, Vancouver BC V6T 1Z2, Canada

School of Mathematics, University of Minnesota, 206 Church Street SE, Minneapolis, MN 55455, United States

Department of Mathematics, University of British Columbia, 1984 Mathematics Road, Vancouver BC V6T 1Z2, Canada

Department of Mathematics, University of California, Berkeley, 970 Evans Hall, Berkeley, CA 94720, United States 


\section{Algebra \& Number Theory}

msp.org/ant

\section{EDITORS}

MANAGING EDITOR

Bjorn Poonen

Massachusetts Institute of Technology

Cambridge, USA

\author{
EDITORIAL BOARD CHAIR \\ David Eisenbud \\ University of California \\ Berkeley, USA
}

BOARD OF EDITORS

Georgia Benkart

Dave Benson

Richard E. Borcherds

John H. Coates

J-L. Colliot-Thélène

Brian D. Conrad

Hélène Esnault

Hubert Flenner

Edward Frenkel

Andrew Granville

Joseph Gubeladze

Roger Heath-Brown

Craig Huneke

Yujiro Kawamata

János Kollár

Yuri Manin

Barry Mazur

Philippe Michel

Susan Montgomery
University of Wisconsin, Madison, USA

University of Aberdeen, Scotland

University of California, Berkeley, USA

University of Cambridge, UK

CNRS, Université Paris-Sud, France

University of Michigan, USA

Freie Universität Berlin, Germany

Ruhr-Universität, Germany

University of California, Berkeley, USA

Université de Montréal, Canada

San Francisco State University, USA

Oxford University, UK

University of Virginia, USA

University of Tokyo, Japan

Princeton University, USA

Northwestern University, USA

Harvard University, USA

École Polytechnique Fédérale de Lausanne

University of Southern California, USA
Shigefumi Mori

Raman Parimala

Jonathan Pila

Anand Pillay

Victor Reiner

Peter Sarnak

Joseph H. Silverman

Michael Singer

Vasudevan Srinivas

J. Toby Stafford

Bernd Sturmfels

Richard Taylor

Ravi Vakil

Michel van den Bergh

Marie-France Vignéras

Kei-Ichi Watanabe

Efim Zelmanov

Shou-Wu Zhang
RIMS, Kyoto University, Japan

Emory University, USA

University of Oxford, UK

University of Notre Dame, USA

University of Minnesota, USA

Princeton University, USA

Brown University, USA

North Carolina State University, USA

Tata Inst. of Fund. Research, India

University of Michigan, USA

University of California, Berkeley, USA

Harvard University, USA

Stanford University, USA

Hasselt University, Belgium

Université Paris VII, France

Nihon University, Japan

University of California, San Diego, USA

Princeton University, USA

\section{PRODUCTION}

production@msp.org

Silvio Levy, Scientific Editor

See inside back cover or msp.org/ant for submission instructions.

The subscription price for 2014 is US $\$ 225 /$ year for the electronic version, and $\$ 400 /$ year ( $\$ 55$, if shipping outside the US) for print and electronic. Subscriptions, requests for back issues and changes of subscribers address should be sent to MSP.

Algebra \& Number Theory (ISSN 1944-7833 electronic, 1937-0652 printed) at Mathematical Sciences Publishers, 798 Evans Hall \#3840, c/o University of California, Berkeley, CA 94720-3840 is published continuously online. Periodical rate postage paid at Berkeley, CA 94704, and additional mailing offices.

ANT peer review and production are managed by EditFLOw ${ }^{\circledR}$ from Mathematical Sciences Publishers.

\section{PUBLISHED BY}

mathematical sciences publishers

nonprofit scientific publishing

http://msp.org/

(C) 2014 Mathematical Sciences Publishers 


\section{Algebra \& Number Theory}

Volume $8 \quad$ No. $4 \quad 2014$

The derived moduli space of stable sheaves

Kai Behrend, Ionut Ciocan-Fontanine, Junho Hwang and Michael Rose

Averages of the number of points on elliptic curves

Greg Martin, Paul Pollack and Ethan Smith

Noncrossed product bounds over Henselian fields

Timo Hanke, DanNy NeFtin and JaCk SONN

Yangians and quantizations of slices in the affine Grassmannian

Joel Kamnitzer, Ben Webster, Alex Weekes and Oded Yacobi

Equidistribution of values of linear forms on quadratic surfaces

OLIVER SARGENT

Posets, tensor products and Schur positivity

Vyjayanthi Chari, Ghislain Fourier and Daisuke SAGaKi

Parameterizing tropical curves I: Curves of genus zero and one

DAVID E. SPEYER

Pair correlation of angles between reciprocal geodesics on the modular surface

Florin P. Boca, Vicențiu Pașol, AleXandru A. Popa and AleXandru

ZAHARESCU

Étale contractible varieties in positive characteristic

ARMin HolschbaCh, JohanNes SCHMIDT and JaKob STIX 